\title{
Evaluation of agronomic performances of rainfed barley double- haploids (DHs) lines under semi-arid conditions
}

\author{
LARBI KARKOUR ${ }^{1,2, \boldsymbol{v}}$, MOHAMMED FENNI ${ }^{3}$, DALILA RAMLA, DJOUHER GAAD ${ }^{2}$, \\ ABDELKADER BENBELKACEM ${ }^{5}$ \\ ${ }^{1}$ Department of Agronomy, Faculty of Life and Natural Sciences, Ferhat Abbas University. Setif-1, 19000 Setif, Algeria \\ Tel.: +213-71-91-85-66. `email: 1.karkour@crbt.dz \\ ${ }^{2}$ Division of Agriculture and Biotechnology, National Research Center for Biotechnology. Constantine, Algeria \\ ${ }^{3}$ Valorization of Natural Biological Resources Laboratory, Faculty of Life and Natural Sciences, Ferhat Abbas University. Setif-1, 19000 Setif, Algeria \\ ${ }^{4}$ National Agronomic Research Institute of Algeria (INRAA). BP 37, Baraki, Alger, Algeria \\ ${ }^{5}$ Plant Breeding and Biotechnology Division, National Agronomic Research Institute of Algeria (INRAA). 25000 Constantine, Algeria
}

Manuscript received: 9 April 2019. Revision accepted: 27 April 2019.

\begin{abstract}
Karkour L, Fenni M, Ramla D, Gaad D, Benbelkacem A. 2019. Evaluation of agronomic performances of rainfed barley double-haploids (DHs) lines under semi-arid conditions. Biodiversitas 20: 1398-1408. Sixty-six doubled-haploid (DHs) barley lines derived from F2 plants of three bi-parental single crosses, between the local variety (Tichdrett) and three introduced genotypes (Express, Plaisant, and Exito) were evaluated in 2017 for agronomic performance. The experiment was undertaken at two locations under rainfed conditions in semi-arid zone of Algeria in a randomized block design with three replications. Each line was scored for fifteen quantitative traits: number of days to heading, thousand grains weight, number of grains per spike, spikes number per $\mathrm{m}^{2}$, plant height, spike length, leaf relative water content, awn length, peduncle length, spike weight, grain weight per spike, leaf area, specific leaf weight, grain yield and harvest index. The results indicated that the presence of highly significant genotype effect for all traits except for leaf area and highly significant environment effect for all characters, except spikes number per $\mathrm{m}^{2}$ and awns length. Genotype $\mathrm{x}$ environment interaction was highly significant for all traits, except specific leaf weight and leaf area. Significant positive correlation between the twelve agronomic traits, ranging from 0.45 to 0.87 , were observed among the number of grains per spike, thousand grains weight, the number of spikes per $\mathrm{m}^{2}$, yield of grains and harvest index. The principal component analysis showed that four components could describe $72 \%$ of total variances. Cluster analysis divided all genotypes studied into three cluster groups. Overall, results of agronomic parameters and those related to the biotic and abiotic stresses will be used for better identifying this germplasm and betterdirecting studies of genetic improvement.
\end{abstract}

Keywords: Cluster analysis, Hordeum vulgare, quantitative traits, Tichedrett, yield components

\section{INTRODUCTION}

Barley (Hordeum vulgare L.), is one of the first domesticated cereals, which contributes from approximately $6 \%$ global cereals and $11.5-12 \%$ of the coarse cereals production (Pal et al. 2012; Kumar et al. 2013b; Kumar et al. 2014). Barley is used as food crop in many countries of Africa, Middle East, South America, and Asian. During 2013, barley was grown on 49.14 million hectares with a production of 143 million metric tonnes (FAOSTAT 2015). In Algeria, barley is the second most important rainfed cereal after durum wheat (Triticum durum Desf.) with 1 million ha harvested annually (Ramla et al. 2017). Its production is mainly located in semi-arid, highland climatic zones $(300-400 \mathrm{~mm}$ rainfall) characterized by highly variable and severe of climate conditions (irregular quantity and distribution of rainfall, spring frost, low winter temperatures and high temperatures of end growing period. These harsh climatic conditions have a negative impact on the level and stability of grain yields which varied between $11.0 \mathrm{q} \mathrm{ha}^{-1}$ in dry years and 27 $\mathrm{q} \mathrm{ha}{ }^{-1}$ in rainy years (MADR 2014). For a long time, the national food security program focused on grain yield as criteria of selection. However, this strategy was conditioned by favorable and stable conditions (Ceccarelli 1996). Consequently, few varieties have been released and released varieties have poor adaptation and low stability. Therefore, they have not been adopted by the farmers. Only two varieties, Tichedrett, and Saida, selected from within local germplasm, remain widely used and cover the major areas occupied by this crop (Ceccarelli et al. 2011; RahalBouziane et al. 2015). In regions with unfavorable cultivation conditions, the creation of genetic gain varieties is an important research objective (Ceccarelli and Impiglia 1998). Obtaining these varieties is, however, conditioned by the use of local germplasm and by the implementation of the selection process in the environment for which these new varieties are intended (Ceccarelli 1996; Ceccarelli et al. 2004). Compared to conventional breeding, plant biotechnologies, and more precisely haploid production techniques, are a powerful tool for rapidly obtaining new pure lines from hybrids, thus simplifying and shortening the selection cycle (Ma et al. 1999; Gomez-Pando et al. 2009).

The adaptability of a variety of diverse environments is usually tested by the degree of its interaction with different 
environments under which it is grown. Particular genotypes normally demonstrate their full genetic potential only under optimum environmental conditions. Therefore, relative performance in a set of varieties should be evaluated over a series of environments. Unstable varieties are those that show significant fluctuations in agronomic performance across different environments which is due to the presence of Genotype $\times$ Environment interactions (GEI) (Alberts 2004). Because of their importance in plant breeding and evolution, $\mathrm{G} \times \mathrm{E}$ of quantitative traits have been the subject of extensive investigations for several crops including barley (Teulat et al. 2001; Pillen et al. 2003; Peighambari et al. 2005; Sameri et al. 2006; Shahinnia et al. 2006; Von Korff et al. 2006).

The objectives of this study are to evaluate doubledhaploid (DHs) barley lines that are derived from the crossing between the local variety Tichedrett and the three introduced lines: Express, Plaisant and Exito, for their agronomic characteristics and to analyze their agronomic performance in two locations under rainfed conditions of Algerian semi-arid zones.

\section{MATERIALS AND METHODS}

\section{Plant materials}

Plant material consisted of sixty-six (66) doubledhaploid (DHs) along with fourth six-row barley parental genotypes (Table 1).These double-haploids derived from F2 plants of 3 bi-parental single crosses between the local variety (Tichedrett) and three introduced genotypes (Express, Plaisant, and Exito) using anther culture as described by Jacquard et al. (2006) cited by Ramla et al. (2017). Tichedrett is characterized by drought resistant and low yielding (Khaldoun et al. 1990), and the three genotypes Express, Plaisant and Exito are high yieldings and susceptible to drought stress (Teulat-Merah et al. 1998). The cross was carried out at the National Agronomic Research Institute of Algeria (INRAA).

\section{Experimental design}

These materials were evaluated at two locations in 2017/2018 year, under rainfed conditions in the Algerian semi-arid region. The locations were: the field Crop Institute of Agricultural Experimental Station of Setif (ITGC-AES) at $979 \mathrm{~m}$ above sea level with $334.7 \mathrm{~mm}$ precipitations and the second locations was: Crop InstituteAgricultural Station of EL-Khroub (ITGC-AES), Constantine at $584 \mathrm{~m}$ altitude with $403 \mathrm{~mm}$ rainfall. Details of the geographic and climatic conditions of trials conditions are presented in Table 2. Field experiments were conducted at each location in a randomized complete block design with three replications. The experimental plots were $2.5 \mathrm{~m}$ long and $1.20 \mathrm{~m}$ wide with $20 \mathrm{~cm}$ between rows. The seeding rate was 270 seeds $\mathrm{m}^{2}$. Sowing was performed on $11 / 12 / 2017$ and 29/12/2017 at the Setif and Constantine station, respectively. Recommended cultural practices for the area were followed.
Table1. Name and code of tested genotypes (parental varieties and double-haploid lines)

\begin{tabular}{|c|c|}
\hline Genotypes/Lines & Code \\
\hline Tichedrett & $\mathrm{Ti}$ \\
\hline Express & EXP \\
\hline Plaisant & PLAI \\
\hline Exito & EXI \\
\hline DH3 F2 Tichedrett*express & HD3 \\
\hline DH4 F2 Tichedrett*express & HD4 \\
\hline DH8 F2 Tichedrett*express & HD8 \\
\hline DH12 F2 Tichedrett*express & HD12 \\
\hline DH18 F2 Tichedrett*express & HD18 \\
\hline DH19 F2 Tichedrett*express & HD19 \\
\hline DH10 F2 Tichedrett*express & HD10 \\
\hline DH22 F2 Tichedrett*express & HD22 \\
\hline DH23 F2 Tichedrett*express & HD23 \\
\hline DH27 F2 Tichedrett*express & HD27 \\
\hline DH28 F2 Tichedrett*express & HD28 \\
\hline DH11 F2 Tichedrett*express & HD11 \\
\hline DH14 F2 Tichedrett*express & HD14 \\
\hline DH15 F2 Tichedrett*express & HD15 \\
\hline DH34 F2 Tichedrett*express & HD34 \\
\hline DH35 F2 Tichedrett*express & HD35 \\
\hline DH38 F2 Tichedrett*express & HD38 \\
\hline DH21 F2 Tichedrett*express & HD21 \\
\hline DH44 F2 Tichedrett*express & HD44 \\
\hline DH47 F2 Tichedrett*express & HD45 \\
\hline DH47 F2 Tichedrett*express & HD47 \\
\hline DH48 F2 Tichedrett*express & HD48 \\
\hline DH49 F2 Tichedrett*express & HD49 \\
\hline DH131 F1Tichedrett*express & HD131 \\
\hline DH130 F1Tichedrett*express & HD130 \\
\hline DH52 F2 Tichedrett*express & HD52 \\
\hline DH94 F1Tichedrett*express & HD94 \\
\hline DH95 F1Tichedrett*express & HD95 \\
\hline DH96 F1Tichedrett*express & HD96 \\
\hline DH98 F1Tichedrett*express & HD98 \\
\hline DH99 F1Tichedrett*express & HD99 \\
\hline DH104 F1Tichedrett*express & HD104 \\
\hline DH106 F1Tichedrett*express & HD106 \\
\hline DH108 F1Tichedrett*express & HD108 \\
\hline DH110 F1Tichedrett*express & HD110 \\
\hline DH112 F1Tichedrett*express & HD112 \\
\hline DH113 F1Tichedrett*express & HD113 \\
\hline DH114F1Tichedrett*express & HD114 \\
\hline DH116 F1Tichedrett*express & HD116 \\
\hline DH117 F1Tichedrett*express & HD117 \\
\hline DH119 F1Tichedrett*express & HD119 \\
\hline DH120 F1Tichedrett*express & HD120 \\
\hline DH122 F1Tichedrett*express & HD122 \\
\hline DH125 F1Tichedrett*express & HD125 \\
\hline DH126 F1Tichedrett*express & HD126 \\
\hline DH127 F1Tichedrett*express & HD127 \\
\hline DH132 F1Tichedrett*express & HD132 \\
\hline DH133 F1Tichedrett*express & HD133 \\
\hline DH134 F1Tichedrett*express & HD134 \\
\hline DH26F2 Tichedrett*express & HD26 \\
\hline DH30 F2 Tichedrett*express & HD30 \\
\hline DH39 F2 Tichedrett*express & HD39 \\
\hline DH38 F2 Tichedrett*express & HD38 \\
\hline DH46 F2 Tichedrett*express & HD46 \\
\hline DH62 F2 Tichedrett*express & HD62 \\
\hline DH84 F2 Tichedrett*express & HD84 \\
\hline DH85 F2 Tichedrett*express & HD85 \\
\hline DH89 F2 Tichedrett*express & HD89 \\
\hline DH90 F2 Tichedrett*express & HD90 \\
\hline DH91 F2 Tichedrett * plaisant & HD91 \\
\hline DH92 F2 Tichedrett * plaisant & HD92 \\
\hline DH74 F1Tichedrett*Exito & HD74 \\
\hline DH77 F2Tichedrett*Exito & HD77 \\
\hline DH80 F2Tichedrett*Exito & HD80 \\
\hline DH82 F2Tichedrett*Exito & HD82 \\
\hline DH135 F1Tichedrett*Exito & HD135 \\
\hline
\end{tabular}


Table 2. Geographic coordinates and climatic characteristics of the two-field trial locations

\begin{tabular}{lll}
\hline \multirow{2}{*}{ Location characteristics } & \multicolumn{2}{c}{ Experimental sites } \\
\cline { 2 - 3 } & \multicolumn{1}{c}{$\begin{array}{c}\text { Institute-Agricultural Experimental } \\
\text { Station of EL-Khroub, (ITGC-AES) }\end{array}$} & \multicolumn{1}{c}{$\begin{array}{c}\text { Institute-Agricultural Experimental Station of } \\
\text { Setif (ITGC-AES) }\end{array}$} \\
\hline Geographic coordinate & High plains & Highlands \\
Latitude & $36^{\circ} 29^{\prime} \mathrm{N}$ & $36^{\circ} 10^{\prime} \mathrm{N}$ \\
Longitude & $6^{\circ} 41^{\prime} \mathrm{E}$ & $521^{\prime} \mathrm{E}$ \\
Altitude & 584 & 979 \\
Climate type & Mediterranean type, Semi-arid & Mediterranean type, continental, Semi-arid \\
Rainfall (Novembre-June) mm & 403 & 334.7 \\
Temperature Minimal $\left({ }^{\circ} \mathrm{C}\right)$ & 8.7 & 6.87 \\
Temperature Maximal $\left({ }^{\circ} \mathrm{C}\right)$ & 20.4 & 18.48 \\
Soil characteristics & clay-loam with clay varies from 30 to 39\% & silt-clay soil with calcium carbonate and organic \\
& and organic matter $0.95-1.25 \%$, rich in & matter contents of 30.4 $\%$ and 1.4\%, respectively \\
& limestone with a content of 12\% (Derbal & (Kribaa et al. 2001; Chennafi et al. 2008). \\
\hline
\end{tabular}

\section{Notation and measurements}

DHs lines were evaluated for the fifteen agronomic traits: Number of days to heading (DHE) was recorded as the number of calendar days from the date of sowing to the time when $50 \%$ of the spikes were halfway out from the flag leaf. At the heading stage, four leaves were sampled per plot from each DH line and parents to measure leaf relative water content (RWC), leaf area (LA) and specific leaf weight (SLW). The leaf area was estimated using the method described by Bekherchouche et al. (2009). Specific leaf weight was calculated according to the following formulae: SLW= FW/LA, where FW is the sample fresh weight and LA is the leaf area (Araus et al. 1998).

The same leaf samples were used to measure leaf relative water content (RWC) using Barrs and Weartherly (1962) method described by Pask et al. (2012). At maturity, Plant height $(\mathrm{PH})$ was calculated as the average height of five randomly selected plants per line measured from the ground to the top of the terminal spikelet (excluding awns). Ten consecutive plants were randomly collected from each plot were used to calculate: spike length (SL); number of grains per spike (NGS); grain weight per spike (GWS); peduncle length (PL); awns length (AL) and spike weight (SW). Spikes number per $\mathrm{m}^{2}(\mathrm{SN})$ and grain yield (GY) were recorded from a vegetative sample harvested from one row, $1.0 \mathrm{~m}$ long per plot. Harvest index (HI) was derived as 100 times the ratio of grain yield to above ground biomass: $\mathrm{HI}=100 \times \mathrm{GY} / \mathrm{BIO}$. Thousand grains weight (TGW) was determined as the weight of a sample of 250 grains after harvest.

\section{Statistical methods}

PROC GLM within Statistical Analysis System (SAS) version 9 was used for the statistical analyses of quantitative data (SAS, 2015) For each Location and for each trait, mean \pm standard deviation, coefficients of variation, and treatment means were compared statistically using Student-Newman-Keuls test at $\mathrm{p}=0.05$. Pearson's correlation coefficient between traits was also calculated. Principal Component Analysis (PCA) was performed in order to identify the most discriminant quantitative and traits. The nearest neighbor option based on Euclidean distances was used to explore relationships among the accessions, which were performed using Ward's minimum variance method. PCA and cluster analysis were performed with XLSTAT v13.01 software (Addin Soft, New York, USA). Furthermore, significance was determined a priori at alpha $=0.05$ probability.

\section{RESULTS AND DISCUSSION}

The summary statistics of the phenotypic performance of the $66 \mathrm{DHs}$ lines and 4 parents for 15 agronomic traits assessed in two each location are shown in Table3. The mean phenotypic values of the twelve significant characters for both locations are reported in Table 4 .

\section{Single location}

Analysis of variance across environments indicated the presence of highly significant genotype effect for all traits except leaf area (LA) and a highly significant environment effect for all characters studied, except spikes number per $\mathrm{m}^{2}(\mathrm{SN})$ and Awns length (AL). The effect for location was height significant for harvest index. HI was earlier at Setif location (mean 29.8\%) with coefficients of variation (CV) (38\%), and late at Constantine location (mean 19.54\%), with CV (30\%). If average data of the two locations are considered, line HD10 (28.88\%), HD39 (25.30\%) and HD11 (24.77\%) with the lowest Harvest index at Constantine, and line HD11 (59.54\%), HD10 (42.21\%), and HD126 (42.15\%) that has showed the highest harvest index at Setif.

Location effect is very significant $(p<0.001)$ for number of days to heading (DHE). At Constantine location, the days to heading ranged between 106 and 121 days after sowing (DAS) with a mean of 112 DAS. The lines HD39, HD10, HD11, HD14, and HD46 were the earliest for number of days to heading (107 at 109 DAS). Whereas, the locale variety Tichedrett (Ti) was later in days to heading 
(mean 112DAS). At Setif location, days to heading was greater ranged between 125 and 135 DAS with a mean of 132 DAS. The lines HD38, HD11, HD80, HD92, and HD91 were the earliest for days to heading (128 at 129DAS) and the lines HD10, HD126 was later in days to heading (130 DAS).

Highly significant differences have existed between various barley genotypes in thousand grains weight (TGW). Lines HD11, HD90. HD10, HD81, and HD26 at Setif location and lines HD39, HD38, HD26 and HD112 at Constantine location gave the highest values for thousand grains weight. Variation was observed for number of grains per spike over the two locations. Lines HD104, HD62, HD48, HD52, HD12, HD84, and HD92 recorded the highest number of grains per spike at Constantine. At Setif location the highest number of grains per spike was recorded by the lines HD84, HD39, HD110, HD98, HD130, and HD92.

The grain yield (GY) was influenced by location. It was higher at Constantine (70.1 Qx.ha ${ }^{-1}$ ) compared to the Setif. Significant $(\mathrm{P}<0.001)$ grain yield differences were observed among lines of barley for both locations. The lines HD116, HD91, HD62, HD38, HD90, and HD11, had the highest grains yield at Constantine; whereas, the lines HD84, HD39, HD110, HD98, HD130, and HD92 had the highest grain yields at Setif location.

At Constantine location the highest Spike weight was produced by the linesHD26 (5.64g), HD45 (3.87g) followed by HD52 (4.16g). At Setif, the highest Spike weight was $3.13 \mathrm{~g}, 2.93 \mathrm{~g}, 2.88 \mathrm{~g}$, and $2.56 \mathrm{~g}$ obtained by the HD96, HD135, HD94, and HD30 respectively. At Setif location the tallest lines were at Setif location HD15 $(97.67 \mathrm{~cm})$, HD133 $(97.00 \mathrm{~cm})$ followed by HD44 $(96.00 \mathrm{~cm})$; however, genotype express produced minimum plants height $(78.67 \mathrm{~cm})$. At Constantine, maximum plant heights were $111.00,109.67,108.00$ and $107.67 \mathrm{~cm}$, obtained by HD62, HD90, HD119, and HD44 respectively.

Variation was observed for peduncle length over the two locations. The lines HD11, HD125, HD38, and HD62 recorded the longest peduncles measuring 28.33, 28.33, 27.67 and $27.33 \mathrm{~cm}$ respectively at Setif. The shortest peduncle length was recorded for Exito $(21.00 \mathrm{~cm})$. At Constantine the longest peduncle lengths were 32.67, 34.33 and $31.00 \mathrm{~cm}$ for HD84, HD117 and Plaisant respectively, the shortest was recorded for HD116 $(21.67 \mathrm{~cm})$.

\section{Combined locations}

Among the traits reported here, except the specific weight leaf and Awns length, days to heading and plant height manifested the lowest variability with a coefficient of variation of $8.53 \%$ and $9.69 \%$, respectively, followed by peduncle length, spike length, thousand-grain weight and grain weight per spike. At the same time, grain yield and yield components were the most variable traits with coefficients of variation ranging from $12.37 \%$ for thousand grains weight to $17.46 \%$ for number of grains per spike. Number of days to heading was influenced by genotype location. Lines HD38, HD91, and HD90 are early with flowering dates $116.3,117.3$ and 117.2 days respectively. The latest flowering was the introduction of genotype (Plaisant) at 126 days.

A significant genotype $\mathrm{x}$ location interaction for Harvest index. The lines HD11, HD10, and HD39 with 58. 38.16 and $36.34 \%$ respectively, gave the highest value for harvest index while HD49 had the lowest harvest index at $15.32 \%$.

Table 3. Statistical data recorded with fifteen agronomic traits for sixty-six double-haploid (DH) barley lines and four parents evaluated in two locations (Constantine and Setif)

\begin{tabular}{|c|c|c|c|c|c|c|c|c|c|c|c|c|c|}
\hline \multicolumn{6}{|c|}{ Mean squares } & \multicolumn{8}{|c|}{ Mean Min. and Max. values and coefficients of variation (\%) } \\
\hline SV & Block & Loc & Gen & Loc*Gen & Resi & Consta & tine lo & cation & & & f locat & ion & \\
\hline DF & $\mathbf{2}$ & 1 & 69 & 69 & 138 & Mean \pm SD & Min & Max & CV & Mean \pm SD & Min & Max & CV \\
\hline$\overline{\mathrm{HI}}$ & 9.06 & $11126^{* * * *}$ & $226.76^{\text {**** }}$ & $142.50^{* * * *}$ & 32.70 & $19.54 \pm 5.81$ & 6.24 & 51.5 & 30 & $29.8 \pm 11.48$ & 9.76 & 60 & 38 \\
\hline TGW & 0.23 & $158.35^{* * * *}$ & $164.90^{* * *}$ & $16.29^{* * *}$ & 0.44 & $45.27 \pm 8.48$ & 30.2 & 56.5 & 12 & $44 \pm 5.651$ & 29.3 & 55.4 & 13 \\
\hline NGS & 5.95 & $5378.59^{* * *}$ & $183.77^{\text {**** }}$ & $100.84^{* * *}$ & 8.17 & $49.8 \pm 15.66$ & 35 & 78 & 16 & $42.6 \pm 6.638$ & 25 & 58 & 16 \\
\hline GY & 65.08 & $17051.3^{* * * *}$ & $316.31^{* * * *}$ & $114.25^{* * *}$ & 30.13 & $40.13 \pm 11.73$ & 11.1 & 70.1 & 29 & $30.5 \pm 11.37$ & 11 & 61.6 & 35 \\
\hline $\mathrm{SN}$ & 7740.3 & $9737.61^{\mathrm{ns}}$ & $9241.77^{* * * *}$ & $4533.7^{* * *}$ & 1927.4 & $183.6 \pm 61.83$ & 40.6 & 391 & 34 & $174 \pm 58.41$ & 50 & 376 & 34 \\
\hline RWC & 109.5 & $45005.1^{* * *}$ & $308.37^{\text {**** }}$ & $208.12^{* * *}$ & 70.72 & $84.09 \pm 9.694$ & 33.5 & 97.2 & 12 & $63.4 \pm 13.11$ & 15.6 & 89.4 & 21 \\
\hline PH & 91.8 & $10510^{* * * *}$ & $160.37^{* * * *}$ & $125.11^{* * * *}$ & 20.27 & $101.4 \pm 8.104$ & 75 & 124 & 8 & $91.4 \pm 7.511$ & 70 & 107 & 8 \\
\hline SL & 1.02 & $30.56^{* * * *}$ & $5.83^{* * *}$ & $3.16^{* * *}$ & 0.40 & $5.696 \pm 1.405$ & 3 & 10 & 25 & $5.16 \pm 1.244$ & 3 & 9 & 24 \\
\hline $\mathrm{AL}$ & 1,04 & $2.95^{\mathrm{ns}}$ & $9.08^{* * *}$ & $7.33^{* * *}$ & 0.80 & $13.23 \pm 1$ & 10 & 17 & 12 & 011 & 10 & 24 & 15 \\
\hline PL & 8.06 & $239.26^{\text {**** }}$ & $55.09^{* * *}$ & $41.30^{* * * *}$ & 4.97 & $29.02 \pm 4$ & 18 & 40 & 16 & 991 & 15 & 36 & 16 \\
\hline SW & 1.32 & $80.58^{* * *}$ & $1.03^{* * *}$ & $0.53^{* * *}$ & 0.21 & $2.978 \pm 0.79$ & 1.47 & 9.39 & 27 & $2.2 \pm 0.444$ & 1.04 & 3.54 & 21 \\
\hline GWS & 0.31 & $56.49^{* * * *}$ & $0.81^{* * * *}$ & $0.39^{* * * *}$ & 0.09 & $2.46 \pm 0.62$ & 1.02 & 5.5 & 25 & $1.73 \pm 0.376$ & 0.87 & 3.16 & 22 \\
\hline LA & 50.6 & $506.73^{* * * *}$ & $22.15^{\mathrm{ns}}$ & $17.52^{\mathrm{ns}}$ & 17.05 & $8.143 \pm 5.44$ & 1.39 & 75.7 & 67 & $5.94 \pm 2.575$ & 0.5 & 14.7 & 43 \\
\hline SLW & $31.10^{-3}$ & $32.10^{-3 \mathrm{~ns}}$ & $30.10^{-3 \mathrm{~ns}}$ & $34.10^{-3 \mathrm{~ns}}$ & $35.10^{-3}$ & $0.017 \pm 0.13$ & 0.01 & 0.18 & 75 & $0.02 \pm 0.018$ & 001 & 0.2 & 88 \\
\hline DHE & 0.37 & $42280.4^{* * * *}$ & $29.65^{* * *}$ & $13.08^{* * *}$ & 0.80 & $112 \pm 3.27$ & 106 & 121 & 3 & $132 \pm 2.112$ & 125 & 135 & 2 \\
\hline
\end{tabular}

Note: DF: degrees of freedom, SV: Source of variation, Loc: location, Gen: Genotype, Resi: Residual. $* * *$ Significant at $\mathrm{p}_{-}<0.001$, NS $=$ Non-significant at $p_{-}<0.05$. HI: Harvest index, TGW: Thousand grains weight, NGS: Number of grains per spike, GY: Grain yield, SN: Spike number per $\mathrm{m}^{2}$ RWC: leaf relative water content, PH: Plant height, SL: Spike length, AL: Awns length, PL: peduncle length, SW: Spike weight, GWS: grain weight per spike, LA: leaf area, SLW: specific leaf weight, DHE: Number of days to heading. 
Plants height (PHT) varied significantly ( $\mathrm{p}<0.001)$ among genotypes and across locations. The maximum height was attained by the lines HD89 $(108.5 \mathrm{~cm})$, HD133 $(106.3 \mathrm{~cm})$ followed by another line HD85 with $105.2 \mathrm{~cm}$, while the shortest line was HD28 $(77.66 \mathrm{~cm})$ and the genotype Exito $(84 \mathrm{~cm})$. It was observed that the thousandgrain weight (TGW) differed between accessions for genotype $\mathrm{x}$ location. The grand mean was $44.65 \mathrm{~g}$ and mean values varied from 55.92 to $34.08 \mathrm{~g}$. The heaviest weight was detected in the lines HD10 (55.95g), HD39 $(54.17 \mathrm{~g})$ and HD26 (54.15g), the lightest weight $(34.08 \mathrm{~g})$ was the line HD35. The lines HD62 and HD11 had the great value for spike length with 8.1 and $7.3 \mathrm{~cm}$ respectively. The lowest value was recorded for HD92 about $3.5 \mathrm{~cm}$. For the character of peduncle length, the lines HD90 and HD89 had the greatest value by 34.33 and 34.16 $\mathrm{cm}$. Signification variation for leaf relative water content (RCW) detected for genotype $\mathrm{x}$, it varied from52.54 to $86.07 \%$ the height value for was observed in two lines HD38 (86.07 mg.cm ${ }^{-2)}$ followed by HD84 (85\%) and the light value was recorded in the line HD3 (52.54\%).

The Grain yield (GY) was influenced by genotype $x$ location interaction, the grand mean was 36.92 Qx.ha $^{-1}$ and mean values varied from 22.43 to 57.27 Qx.ha ${ }^{-1}$. The heaviest value of grain yield was detected in the lines HD10 (57.27 Qx.ha' ${ }^{-1}$ ), HD11 (55.12Qx.ha ${ }^{-1}$ ) and HD30 (50.03Qx.ha-1). The lightest value of grain yield (22.43Qx.ha ${ }^{-1}$ ) was the line HD80. Number of grain per spike differed among genotype $\mathrm{x}$ location interaction, ranged from 32.8 to 56.83 grains with an overage mean of 46.21 grains. The lines HD84, HD98, and HD62 had greatest number of grains per spike with $56.83,56.5$ and 56 grains respectively. The line Hd116 had the least of NGS (32.83 grains).

Variation was observed for grain weight per spike (GWS) over two, the lines HD15 (3.18g), HD52 (3.01g) and HD92 $(2.72 \mathrm{~g})$ recorded the highest grain weight per spike. The minimum number was also obtained by HD28 (1.21g).

\section{Correlation between characters}

The correlation coefficients between 70 barley genotypes including 66 double-haploid (DHs) and their four parents (Hordeum vulgare) evaluated in two locations are presented in Table 5.

Harvest index was highly significant and positive correlation with grain yield $(\mathrm{r}=0.741, \mathrm{p}<0.001)$, spikes number $(\mathrm{r}=0.567, \mathrm{p}<0.001)$ and thousand grains weight $(r=0.366, p<0.01)$. However, HI was negatively correlated with number of days to heading $(\mathrm{r}=-0.315, \mathrm{p}<0.01)$. Thousand grain weight is highly significant and correlated positively with grain yield $(\mathrm{r}=0.511, \mathrm{p}<0.001)$, leaf relative water content $(r=0.369, p<0.01)$, spike weight $(r=0.316$, $\mathrm{p}<0.01)$ and grain weight per spike $(r=0.321, \mathrm{p}<0.01)$ Number of grains per spike weight exhibited highly significant and positive correlation with spike weight $(r=0.560, p<0.001)$ and grain weight per spike $(r=0.576$, $\mathrm{p}<0.001)$. Grain yield exhibited highly significant and positive correlation with thousand grain weight $(\mathrm{r}=0.511$, $\mathrm{p}<0.001)$, spike number per $\mathrm{m}^{2}(\mathrm{r}=0.597, \mathrm{p}<0.001)$ and relative leaf water content $(r=0.410, p<0.001)$. However, negative correlation of this character was noted with number of days to heading $(r=-0.465, \mathrm{p}<0.001)$. Peduncle length exhibited highly significant and positive correlation with plant height $(\mathrm{r}=0.405, \mathrm{p}<0.001)$. Spike length exhibited highly significant and negative correlation with grain weight per spike $(r=-0.253, p<0,001)$. Spike weight was highly significant and positively correlated with number of grains per spike $(\mathrm{r}=0.576, \mathrm{p}<0.001)$ and grain weight per spike $(0.875, \mathrm{p}<0.001)$.

\section{Principal compound analysis}

Principal compound analysis (PCA) was performed based on twelve characters. There were four Eigenvalues greater than one, which determined the choice of the four components (Table 6). The PCA exhibited variances of $25.07,23.35,12.13$ and $9.79 \%$, were extracted for the first four principal components and accounts about $72 \%$ of total variation. Harvest index, relative leaf water content, thousand grains weight, grain yield and number of days to heading showed greater loading for variation in the first principal component. For Number of grains per spike, spike length, spike weight, grain weight per spike and spikes number most variation was explained by the second principal component. Variation in the third principal component was mainly due to plant height and peduncle length, while the fourth principal component showed $10 \%$ of total variation with greater loading from spike length and number of grains per spike. In line with the present finding, Bedasa et al. (2015) employed principal component analysis for detecting variation in 49 barley population in which the first four PCs contributed $70.36 \%$ of total variation. Generally, days to $50 \%$ flowering, days to maturity, and number of seeds per spike was the most loading character for the variation among accessions

Based on the 2D graph analysis, five major classes were formed (Figure 1). The first class contained all lines characterized by the highest number of grains per spike, grain weight per spike and spike weight. Class 2 contains primarily the lines belonging with highest value of harvest index, grain yield, and thousand grains weight. The third class contained the lines characterized by the highest value of spike length and spikes number per $\mathrm{m}^{2}$. Class four contained the line characterized by long number of days to heading. The fifth class was characterized by tall Lines and those with long peduncles.

\section{Cluster analysis}

The dendrogram performed by cluster analysis confirmed the PCA results and indicated that the sixty-six double-haploid (DHs) barley lines and four parents could be divided into three major groups (Figure 2). 
Table 4. Mean values, coefficient of variation (\%) for twelve quantitative traits recorded at Constantine and Setif locations

\begin{tabular}{|c|c|c|c|c|c|c|c|c|c|c|c|c|}
\hline Gen. & $\begin{array}{l}\text { HI } \\
(\%)\end{array}$ & $\begin{array}{c}\text { TGW } \\
\text { (g) }\end{array}$ & $\begin{array}{l}\text { NGS } \\
\text { (nbr) }\end{array}$ & $\begin{array}{c}\text { GY } \\
\text { (Qx/ha) }\end{array}$ & $\begin{array}{c}\mathrm{SN} \\
\left(\mathrm{nbr} / \mathbf{m}^{2}\right)\end{array}$ & $\begin{array}{c}\text { RWC } \\
(\%)\end{array}$ & $\begin{array}{l}\text { PH } \\
(\mathbf{c m})\end{array}$ & $\begin{array}{l}\text { SL } \\
\text { (cm) }\end{array}$ & $\begin{array}{l}\mathbf{P L} \\
(\mathbf{c m})\end{array}$ & $\begin{array}{r}\text { SW } \\
\text { (g) }\end{array}$ & $\begin{array}{c}\text { GWS } \\
(\mathrm{g})\end{array}$ & $\begin{array}{l}\text { DHE } \\
\text { (days) }\end{array}$ \\
\hline HD10 & $38.16 \mathrm{~b}$ & $55.95_{\mathrm{a}}$ & $42_{\mathrm{u}-\mathrm{x}}$ & $57.27 \mathrm{a}$ & $244.7_{b-f}$ & $83.57_{\mathrm{a}-\mathrm{d}}$ & $96.6_{\mathrm{k}-\mathrm{x}}$ & $6.33_{\mathrm{e}-1}$ & $25.66_{\mathrm{s}-\mathrm{z}}$ & $2.23_{\mathrm{n}-\mathrm{u}}$ & $1.92_{0-y}$ & $119.83_{\mathrm{yz}}$ \\
\hline HD11 & $58.70_{\mathrm{a}}$ & $53.00_{\mathrm{c}}$ & $41.5_{\mathrm{v}-\mathrm{y}}$ & $55.12_{\mathrm{ab}}$ & $280.34_{\mathrm{ab}}$ & $79.35_{a-j}$ & $97.16_{\mathrm{j}-\mathrm{v}}$ & $7.33 \mathrm{~b}$ & $29.83 \mathrm{f}-\mathrm{m}$ & $2.43 \mathrm{j}-\mathrm{S}$ & $1.97 \mathrm{~m}-\mathrm{x}$ & $119.33 \mathrm{az}$ \\
\hline HD30 & $28.00_{d-i}$ & $50.38_{\mathrm{ef}}$ & $48_{\mathrm{j}-\mathrm{q}}$ & $50.03 \mathrm{bc}$ & $206.95_{\mathrm{d}-1}$ & $79.12_{\mathrm{a}-\mathrm{k}}$ & $93.33_{\mathrm{s}-\mathrm{z}}$ & $6.75_{\mathrm{c}-\mathrm{g}}$ & $24.3 \mathrm{axyz}$ & $2.60_{\mathrm{f}-\mathrm{p}}$ & $2.02 \mathrm{k}-\mathrm{w}$ & $118.5_{\mathrm{ab}}$ \\
\hline HD38 & $29.81_{d-g}$ & 49.24 ghi & $50.83 \mathrm{f}-\mathrm{k}$ & $49.62 \mathrm{bcd}$ & $198.95_{\mathrm{e}-\mathrm{o}}$ & $86.07 \mathrm{a}$ & $96.83_{\mathrm{k}-\mathrm{w}}$ & $6.25 \mathrm{f}-1$ & $28.83 j-p$ & $2.19_{\mathrm{o}-\mathrm{u}}$ & $1.88 \mathrm{p}-\mathrm{z}$ & $116.33 \mathrm{e}$ \\
\hline HD39 & $36.34 \mathrm{bc}$ & $54.17_{\mathrm{b}}$ & $41.16_{\mathrm{v}-\mathrm{y}}$ & $49.19_{b-e}$ & $220.67_{c-h}$ & $80.68 \mathrm{a}-\mathrm{h}$ & $101_{\mathrm{c}-\mathrm{l}}$ & $6.08_{g-n}$ & $29.5_{\mathrm{h}-\mathrm{o}}$ & $1.82_{\mathrm{tuv}}$ & $1.53 \mathrm{abc}$ & $117.33_{\text {cde }}$ \\
\hline HD46 & $25.96_{\mathrm{e}-\mathrm{o}}$ & $34.37_{1}$ & $45.16_{\mathrm{o}-\mathrm{u}}$ & $47.57_{\mathrm{c}-\mathrm{f}}$ & $306.40_{\mathrm{a}}$ & $81.61_{\mathrm{a}-\mathrm{f}}$ & $92.33_{\mathrm{u}-\mathrm{z}}$ & $5.5 \mathrm{~m}-\mathrm{t}$ & $22.3 b$ & $2.03_{\mathrm{r}-\mathrm{v}}$ & $1.48 \mathrm{bc}$ & $120.16_{\mathrm{w}-\mathrm{z}}$ \\
\hline HD14 & $28.47_{\mathrm{d}-\mathrm{i}}$ & $49.57_{\mathrm{gh}}$ & $49.83_{\mathrm{g}-1}$ & $46.92_{\mathrm{c}-\mathrm{g}}$ & 201.14 e-n & $68.30_{\mathrm{n}-\mathrm{v}}$ & $95.83_{\mathrm{m}-\mathrm{y}}$ & $6.58_{c-h}$ & $29_{j-p}$ & $2.32 \mathrm{k}-\mathrm{t}$ & $1.82 \mathrm{q}-\mathrm{z}$ & $120_{\text {xyz }}$ \\
\hline HD26 & $26.81_{d-1}$ & $54.15 \mathrm{~b}$ & $47.83 \mathrm{j}-\mathrm{q}$ & $46.65_{\mathrm{c}-\mathrm{h}}$ & $186.20_{\mathrm{f}-\mathrm{u}}$ & $83.37 \mathrm{a}-\mathrm{e}$ & $92_{\mathrm{w}-\mathrm{z}}$ & $5.16 \mathrm{r}-\mathrm{w}$ & $24.83 \mathrm{v}-\mathrm{z}$ & $3.79 \mathrm{a}$ & $2.42_{c-j}$ & $120.66_{u-y}$ \\
\hline HD62 & $26.39_{d-n}$ & 40.49 b-e & $56 \mathrm{abc}$ & $46.30_{c-h}$ & $205.49_{\mathrm{d}-\mathrm{m}}$ & $84.75_{\mathrm{abc}}$ & $102.33 \mathrm{~b}-\mathrm{i}$ & $8.08 \mathrm{a}$ & $27.16 \mathrm{n}-\mathrm{v}$ & $3.06 \mathrm{c}-\mathrm{h}$ & $2.46 \mathrm{c}-\mathrm{g}$ & $121.16_{\text {s-w }}$ \\
\hline HD89 & $31.7 \mathrm{bcd}$ & $48.4_{\mathrm{j}-\mathrm{m}}$ & $48.16_{\mathrm{j}-\mathrm{p}}$ & $44.52_{\mathrm{c}-\mathrm{i}}$ & $191.11_{\mathrm{f}-\mathrm{s}}$ & $70.54_{\mathrm{i}-\mathrm{t}}$ & $108.5 \mathrm{a}$ & $4.83 \mathrm{t}-\mathrm{z}$ & $34.16_{\mathrm{ab}}$ & $2.31_{1-u}$ & $2.07_{\mathrm{j}-\mathrm{u}}$ & $120_{\text {xyz }}$ \\
\hline $\mathrm{Ti}$ & $21.80_{\mathrm{i}-\mathrm{y}}$ & $49.7_{\mathrm{fgh}}$ & $43.16_{\mathrm{s}-\mathrm{v}}$ & $44.41_{\mathrm{c}-\mathrm{i}}$ & $207.53 \mathrm{~d}-1$ & $76.97_{\mathrm{a}-\mathrm{o}}$ & $99.33_{\mathrm{e}-\mathrm{q}}$ & $3.66 \mathrm{bcd}$ & $25.16_{\mathrm{t}-\mathrm{z}}$ & $2.39_{\mathrm{j}-\mathrm{s}}$ & $2.03_{\mathrm{k}-\mathrm{v}}$ & $121.16_{\mathrm{s}-\mathrm{w}}$ \\
\hline HD94 & $27.37_{\mathrm{d}-\mathrm{j}}$ & $49.6_{\mathrm{gh}}$ & $49_{\mathrm{h}-\mathrm{m}}$ & $44.16_{c-i}$ & $179.02_{\mathrm{f}-\mathrm{w}}$ & $79.64_{a-i}$ & $92.5_{\mathrm{t}-\mathrm{z}}$ & 4.16 $\mathrm{abcdz}$ & $23.33_{\mathrm{abz}}$ & $2.97_{\mathrm{c}-\mathrm{i}}$ & $2.63_{\mathrm{cd}}$ & $122.33 \mathrm{l}-\mathrm{r}$ \\
\hline HD90 & $28.13 \mathrm{~d}-\mathrm{i}$ & $49.38_{\mathrm{gh}}$ & $48.5 \mathrm{i}-\mathrm{n}$ & $43.79_{\mathrm{d}-\mathrm{j}}$ & $175.07_{\mathrm{g}-\mathrm{y}}$ & $82.03 \mathrm{a}-\mathrm{f}$ & $102.66 \mathrm{a}-\mathrm{f}$ & $6.16_{\mathrm{g}-\mathrm{m}}$ & $34.33 \mathrm{a}$ & $2.57_{\mathrm{g}-\mathrm{q}}$ & $2.01_{1-x}$ & $117.33 \mathrm{cde}$ \\
\hline HD91 & $26.3_{d-n}$ & $49.98_{\text {efg }}$ & $44.83_{\mathrm{q}-\mathrm{u}}$ & $43.02 \mathrm{e}-\mathrm{k}$ & $189.13_{\mathrm{f}-\mathrm{t}}$ & $81.91_{\mathrm{a}-\mathrm{f}}$ & $97.5_{\mathrm{i}-\mathrm{u}}$ & $4.6_{\mathrm{u}-\mathrm{z}}$ & $30.83_{c-j}$ & $2.72 \mathrm{~d}-\mathrm{n}$ & $2.12 \mathrm{~g}-\mathrm{t}$ & $117_{\mathrm{de}}$ \\
\hline HD85 & $26.45_{\mathrm{d}-\mathrm{m}}$ & 47.28 nop & $45.16_{\mathrm{o}-\mathrm{t}}$ & $42.63 f-1$ & $193.55_{\mathrm{f}-\mathrm{r}}$ & $78.85_{\mathrm{a}-\mathrm{k}}$ & $105.16_{a b c}$ & $5_{s-y}$ & $33.5_{\mathrm{ab}}$ & $2.60_{\mathrm{f}-\mathrm{p}}$ & $2.10 \mathrm{~h}-\mathrm{t}$ & $122.5_{\mathrm{k}-\mathrm{q}}$ \\
\hline HD84 & $26.49_{\mathrm{d}-\mathrm{m}}$ & $48.35_{\mathrm{j}-\mathrm{m}}$ & $56.83_{a}$ & $42.22_{\mathrm{f}-\mathrm{m}}$ & $154.24_{n-z}$ & $85.00_{\mathrm{ab}}$ & 104.66a-d & $5.33_{\mathrm{o}-\mathrm{u}}$ & $32 \mathrm{a}-\mathrm{h}$ & $2.8_{c-j}$ & $2.35_{\mathrm{d}-1}$ & $120_{\mathrm{xyz}}$ \\
\hline HD126 & $32,88 \mathrm{bcd}$ & $44.11_{\mathrm{vw}}$ & $39.83_{\mathrm{w}-\mathrm{z}}$ & $41.93_{\mathrm{f}-\mathrm{m}}$ & $225.74_{c-f}$ & $74.30_{\mathrm{d}-\mathrm{r}}$ & $103.66_{\mathrm{a}-\mathrm{f}}$ & $5.16_{\mathrm{r}-\mathrm{w}}$ & $32.16_{\mathrm{a}-\mathrm{g}}$ & $2.24_{\mathrm{n}-\mathrm{u}}$ & $1.68_{\mathrm{v}-\mathrm{Z}}$ & $125.16_{a b c}$ \\
\hline HD12 & $30.87_{\mathrm{c}-\mathrm{f}}$ & $38.72 \mathrm{~g}$ & $47.66_{\mathrm{k}-\mathrm{q}}$ & $41.38_{\mathrm{g}-\mathrm{n}}$ & $226.96 \mathrm{c}-\mathrm{f}$ & $78.41_{\mathrm{a}-\mathrm{l}}$ & $104 \mathrm{a}-\mathrm{e}$ & $5.75 \mathrm{k}-\mathrm{r}$ & $30.83_{c-j}$ & $2.73 \mathrm{c}-\mathrm{n}$ & $2.37 \mathrm{c}-\mathrm{k}$ & $124.16_{c-g}$ \\
\hline HD92 & $26.41_{d-n}$ & $50.66 \mathrm{e}$ & $53.5 b-f$ & $40.72_{\mathrm{g}-\mathrm{o}}$ & $150.38_{\mathrm{r}-\mathrm{z}}$ & $78.15_{\mathrm{a}-\mathrm{m}}$ & $88.83_{\mathrm{cde}}$ & $3.5 \mathrm{bcd}$ & $29.16_{\text {i-p }}$ & $3.10_{\mathrm{c}-\mathrm{f}}$ & $2.72 \mathrm{bc}$ & $118_{\mathrm{bcd}}$ \\
\hline HD104 & $27.10_{\mathrm{d}-\mathrm{k}}$ & $45.82_{\mathrm{rst}}$ & $53 \mathrm{c}-\mathrm{g}$ & $40.71_{\mathrm{g}-\mathrm{o}}$ & $175.38 \mathrm{~g}-\mathrm{x}$ & $62.36_{t-v}$ & $104.16_{\mathrm{a}-\mathrm{e}}$ & $4.66_{u-z}$ & $29_{j-p}$ & $2.78 \mathrm{c}-\mathrm{m}$ & $2.31 \mathrm{e}-\mathrm{m}$ & $124 \mathrm{~d}-\mathrm{h}$ \\
\hline HD44 & $29.42 \mathrm{~d}-\mathrm{h}$ & $39.97_{\mathrm{def}}$ & $46.661-\mathrm{r}$ & $40.52 \mathrm{~h}-\mathrm{o}$ & $210.37_{\mathrm{d}-\mathrm{j}}$ & $75.52 \mathrm{~b}-\mathrm{q}$ & $93.83_{\mathrm{r}-\mathrm{z}}$ & $5.5 \mathrm{~m}-\mathrm{t}$ & $26_{\mathrm{r}-\mathrm{y}}$ & $2.23 \mathrm{n}-\mathrm{u}$ & $1.67_{\mathrm{w}-\mathrm{z}}$ & $122.66_{\mathrm{j}-\mathrm{p}}$ \\
\hline HD98 & $25.6 \mathrm{e}-\mathrm{q}$ & $45.34_{\mathrm{tu}}$ & $56.5_{\mathrm{ab}}$ & $40.05_{\mathrm{i}-\mathrm{o}}$ & $148.21_{\mathrm{s}-\mathrm{z}}$ & $71.39_{\mathrm{g}-\mathrm{t}}$ & $96.66_{\mathrm{k}-\mathrm{x}}$ & $3.83 \mathrm{bcd}$ & 23.83 abyz & $3.25 \mathrm{bc}$ & $2.57 \mathrm{cde}$ & $120.66 u-y$ \\
\hline 045 & 25.78 e-p & $49.975_{\mathrm{efg}}$ & $44_{\mathrm{r}-\mathrm{v}}$ & $39.89 \mathrm{i}-\mathrm{p}$ & $183.44_{\mathrm{f}-\mathrm{v}}$ & 77.59 a-n & 95.66 m-y & $5.81_{\mathrm{j}-\mathrm{q}}$ & $27.83_{\mathrm{k}-\mathrm{s}}$ & $2.46_{\mathrm{i}-\mathrm{s}}$ & $2.07_{\mathrm{j}-\mathrm{u}}$ & $121.66_{\mathrm{p}-\mathrm{u}}$ \\
\hline HD52 & $24.89_{\mathrm{f}-\mathrm{t}}$ & $44.70_{\mathrm{uv}}$ & $55.66 \mathrm{abc}$ & $39.85_{\mathrm{i}-\mathrm{p}}$ & $160.24_{\mathrm{k}-\mathrm{z}}$ & $83.88 \mathrm{abc}$ & $93 \mathrm{t}-\mathrm{z}$ & $4.33 \mathrm{axyz}$ & $25.33_{\mathrm{s}-\mathrm{z}}$ & $3.04 \mathrm{c}-\mathrm{h}$ & $3.01 \mathrm{ab}$ & 122.83 i-o \\
\hline HD82 & $28.48_{\text {d-h }}$ & $46.97_{\mathrm{opq}}$ & $53.16_{c-f}$ & $39.68 \mathrm{i}-\mathrm{p}$ & $159.33 \mathrm{l}-\mathrm{z}$ & $65.65_{r-v}$ & $87.66_{\mathrm{de}}$ & $4.83 \mathrm{t}-\mathrm{z}$ & $23.8 \mathrm{abyz}$ & $2.64_{\mathrm{e}-\mathrm{o}}$ & $2.27_{\mathrm{e}-\mathrm{o}}$ & $122.16_{\mathrm{m}-\mathrm{s}}$ \\
\hline HD15 & $30.77_{c-f}$ & $51.78_{\mathrm{d}}$ & $55.33_{\mathrm{a}-\mathrm{d}}$ & $38.95_{\mathrm{i}-\mathrm{p}}$ & $139.39_{\mathrm{t}-\mathrm{z}}$ & $75.81_{b-p}$ & $94.33_{\mathrm{q}-\mathrm{z}}$ & $5.83_{\mathrm{i}-\mathrm{q}}$ & $29.83_{\mathrm{f}-\mathrm{m}}$ & $3.45_{\mathrm{ab}}$ & $3.18 \mathrm{a}$ & $123.83_{\mathrm{e}-\mathrm{i}}$ \\
\hline HD99 & $24.80_{\mathrm{f}-\mathrm{u}}$ & $48.25 \mathrm{klm}$ & $45.83 \mathrm{~m}-\mathrm{s}$ & $38.88_{\mathrm{i}-\mathrm{p}}$ & $164.70_{\mathrm{j}-\mathrm{z}}$ & $73.88_{\mathrm{e}-\mathrm{r}}$ & $97.33_{\mathrm{i}-\mathrm{v}}$ & $4.41_{\mathrm{axyz}}$ & $27.41_{\mathrm{m}-\mathrm{u}}$ & $2.69_{\mathrm{e}-\mathrm{o}}$ & $2.28_{\mathrm{e}-\mathrm{n}}$ & $123.66_{e-j}$ \\
\hline HD131 & $21,66_{i-y}$ & $43.82_{\mathrm{wx}}$ & $52.83_{\mathrm{c}-\mathrm{g}}$ & $38.52 \mathrm{i}-\mathrm{p}$ & $180.06_{\mathrm{f}-\mathrm{w}}$ & $55.87 \mathrm{wx}$ & $93.16_{\mathrm{s}-\mathrm{z}}$ & $5.25_{\mathrm{p}-\mathrm{v}}$ & $25.3_{\mathrm{s}-\mathrm{z}}$ & $3.06_{\mathrm{c}-\mathrm{h}}$ & $2.46_{c-g}$ & $122.16_{\mathrm{m}-\mathrm{s}}$ \\
\hline HD113 & $21.93 \mathrm{i}-\mathrm{x}$ & $39.83_{\mathrm{ef}}$ & $50.66 \mathrm{f}-\mathrm{k}$ & $37.85_{\mathrm{j}-\mathrm{r}}$ & $173.70_{\mathrm{h}-\mathrm{y}}$ & $60.61_{u-v}$ & $91.33_{\mathrm{ayz}}$ & $4.41_{\mathrm{axyz}}$ & $27_{\mathrm{o}-\mathrm{w}}$ & $2.58 \mathrm{f}-\mathrm{q}$ & $2.18 \mathrm{f}-\mathrm{p}$ & $124 \mathrm{~d}-\mathrm{h}$ \\
\hline HD119 & $21.39_{\mathrm{j}-\mathrm{y}}$ & $44.4_{\mathrm{vw}}$ & $37.33_{\mathrm{az}}$ & $37.63_{\mathrm{j}-\mathrm{s}}$ & $224.60_{\mathrm{c}-\mathrm{g}}$ & $74.31_{\mathrm{d}-\mathrm{r}}$ & $100.5_{\mathrm{d}-\mathrm{m}}$ & 4.16 $6_{a b c d z}$ & $28.6_{\mathrm{j}-\mathrm{q}}$ & $2.25 \mathrm{~m}-\mathrm{u}$ & $1.96 \mathrm{~m}-\mathrm{x}$ & $123.5_{\mathrm{f}-\mathrm{k}}$ \\
\hline HD 112 & $25.34 \mathrm{e}-\mathrm{r}$ & 49.10hij & $43.66 \mathrm{r}-\mathrm{v}$ & $37.25 \mathrm{k}-\mathrm{t}$ & $184.43 \mathrm{f}-\mathrm{v}$ & 76.69 a-p & $97.5_{\mathrm{i}-\mathrm{u}}$ & $4.5_{\mathrm{xyz}}$ & $26.83_{\mathrm{p}-\mathrm{x}}$ & $2.66 \mathrm{e}-\mathrm{o}$ & $2.21_{\mathrm{f}-\mathrm{p}}$ & $121.83_{\mathrm{o}-\mathrm{t}}$ \\
\hline HD27 & $25.22_{\mathrm{f}-\mathrm{s}}$ & $46.78_{\mathrm{pqr}}$ & $42.5_{t-x}$ & $37.22 \mathrm{k}-\mathrm{t}$ & $194.62 \mathrm{e}-\mathrm{q}$ & $70.69_{\mathrm{i}-\mathrm{t}}$ & $94.16_{\mathrm{r}-\mathrm{z}}$ & $5.08_{r-x}$ & $26.16_{\mathrm{q}-\mathrm{x}}$ & 3.15 b-e & $2.17_{\mathrm{g}-\mathrm{q}}$ & $121.16_{\mathrm{s}-\mathrm{w}}$ \\
\hline 108 & $24.01_{\mathrm{g}-\mathrm{u}}$ & $40.74 \mathrm{bc}$ & $48.33_{\mathrm{j}-\mathrm{o}}$ & $37.20_{\mathrm{k}-\mathrm{t}}$ & $177.68_{\mathrm{f}-\mathrm{w}}$ & $73.01_{\mathrm{f}-\mathrm{r}}$ & $98.83 \mathrm{f}-\mathrm{r}$ & $6.55_{\mathrm{c}-\mathrm{i}}$ & $31.66 \mathrm{~b}-\mathrm{i}$ & $3.23 \mathrm{bcd}$ & 2.58 cde & $123.66 \mathrm{e}-\mathrm{j}$ \\
\hline HD28 & $25.15_{\mathrm{f}-\mathrm{s}}$ & $35.65_{\mathrm{k}}$ & $39.5_{x y z}$ & $37.19_{\mathrm{k}-\mathrm{t}}$ & $252.31_{\mathrm{bcd}}$ & $67.26_{p-v}$ & $77.66_{\mathrm{f}}$ & $6.58 \mathrm{c}-\mathrm{h}$ & $27.66_{1-t}$ & $1.65_{\mathrm{v}}$ & $1.21_{\mathrm{c}}$ & $122.66_{\mathrm{j}-\mathrm{p}}$ \\
\hline HD95 & $23.5_{\mathrm{g}-\mathrm{v}}$ & $47.85_{\mathrm{lmn}}$ & $43.16_{\mathrm{s}-\mathrm{v}}$ & $37.17_{\mathrm{k}-\mathrm{t}}$ & $175.40_{\mathrm{g}-\mathrm{w}}$ & $71.99_{\mathrm{g}-\mathrm{s}}$ & $97.66_{\mathrm{h}-\mathrm{t}}$ & $4.08 \mathrm{a}-\mathrm{d}$ & $32.83_{\mathrm{a}-\mathrm{e}}$ & $2.43_{\mathrm{j}-\mathrm{s}}$ & $2.06_{\mathrm{k}-\mathrm{u}}$ & $122.5_{\mathrm{k}-\mathrm{q}}$ \\
\hline HD106 & $24.91_{\mathrm{f}-\mathrm{t}}$ & $41.83_{\mathrm{yz}}$ & $44.33_{\mathrm{r}-\mathrm{v}}$ & $36.46_{1-u}$ & $200.74_{e-n}$ & $67.97_{\mathrm{o}-\mathrm{v}}^{\circ}$ & $97.5_{\mathrm{i}-\mathrm{u}}$ & $4.08_{\mathrm{a}-\mathrm{d}}$ & $30.25_{\mathrm{f}-\mathrm{k}}$ & $2.42_{j-s}$ & $2.37_{\mathrm{c}-\mathrm{k}}$ & $123.83_{\mathrm{e}-\mathrm{i}}$ \\
\hline HD127 & & & & $36.16 \mathrm{~m}-\mathrm{u}$ & $.62 \mathrm{~d}-\mathrm{k}$ & $77.74 a-n$ & $101.16_{c-k}$ & $4.8 \mathrm{t}-\mathrm{z}$ & $28.8_{\mathrm{j}-\mathrm{p}}$ & $1.95_{\mathrm{s}-\mathrm{v}}$ & $1.66_{x y z}$ & $123.66_{e-j}$ \\
\hline HD19 & $28.68 \mathrm{~d}-\mathrm{h}$ & $47.19 \mathrm{n}-\mathrm{q}$ & $52.16 \mathrm{e}-\mathrm{h}$ & $36.02 \mathrm{~m}-\mathrm{u}$ & $152.7_{1 \mathrm{n}-\mathrm{z}}$ & $80.82_{\mathrm{a}-\mathrm{g}}$ & $90.66 \mathrm{a}-\mathrm{d}$ & $5.5 \mathrm{~m}-\mathrm{t}$ & $28.8_{\mathrm{j}-\mathrm{p}}$ & $2.86_{c-j}$ & $2.31 \mathrm{e}-\mathrm{m}$ & $122.83_{\mathrm{i}-\mathrm{o}}$ \\
\hline HD3 & $24.68_{\mathrm{f}-\mathrm{u}}$ & $42.12 \mathrm{y}$ & $46.66 \mathrm{l}-\mathrm{r}$ & $35.68 \mathrm{n}-\mathrm{v}$ & $180.08_{\mathrm{f}-\mathrm{v}}$ & $52.54_{x}$ & $95.66_{m-y}$ & $6.58_{c-h}$ & $32.33_{\mathrm{a}-\mathrm{f}}$ & $2.66_{\mathrm{e}-\mathrm{o}}$ & $2.17_{\mathrm{g}-\mathrm{q}}$ & $124.66 \mathrm{~b}-\mathrm{e}$ \\
\hline HD110 & $21.86_{\mathrm{i}-\mathrm{x}}$ & $48.57 \mathrm{i}_{-1}$ & $46 \mathrm{~m}-\mathrm{s}$ & $35.52_{n-v}$ & $141.96_{\mathrm{s}-\mathrm{z}}$ & $62.12 \mathrm{t}-\mathrm{v}$ & $91.5_{\mathrm{y}-\mathrm{z}}$ & $5.08_{\mathrm{r}-\mathrm{x}}$ & $27.83 \mathrm{k}-\mathrm{s}$ & $3.13 \mathrm{~b}-\mathrm{e}$ & $2.53 \mathrm{c}-\mathrm{f}$ & $123.83_{\mathrm{e}-\mathrm{i}}$ \\
\hline HD18 & $19.94_{n-y}$ & $46.43_{\mathrm{qrs}}$ & $55.83_{\mathrm{abc}}$ & 530 & $118.67 \mathrm{abz}$ & $74.17_{\mathrm{d}-\mathrm{r}}$ & $95.33_{\mathrm{n}-\mathrm{z}}$ & $6.41_{\mathrm{d}-\mathrm{k}}$ & $27.83_{\mathrm{k}-\mathrm{s}}$ & $2.66_{\mathrm{e}-\mathrm{o}}$ & $2.13_{\mathrm{g}-\mathrm{s}}$ & $122_{\mathrm{n}-\mathrm{s}}$ \\
\hline & $.94_{\mathrm{h}-\mathrm{x}}$ & & & & $6 \mathrm{k}-\mathrm{z}$ & & & 5.661 & $29_{\mathrm{j}-\mathrm{p}}$ & $2.40_{\mathrm{j}-\mathrm{s}}$ & $2.03 \mathrm{k}-\mathrm{v}$ & $121.5_{\mathrm{q}-\mathrm{v}}$ \\
\hline $\mathrm{HD} 23$ & $19.61_{o-y}$ & $37.022_{j}$ & $39.66_{x-z}$ & $33.76_{p-x}$ & $207.45_{\mathrm{d}-1}$ & $67.30_{\mathrm{p}-\mathrm{v}}$ & $94.33_{\mathrm{q}-\mathrm{z}}$ & $7_{\text {be }}$ & $23 \mathrm{a}-\mathrm{b}$ & $2.06_{\mathrm{q}-\mathrm{v}}$ & $1.51_{b c}$ & $125.16_{a b c}$ \\
\hline HD21 & $18.83_{\mathrm{s}-\mathrm{y}}$ & $46.95_{\mathrm{pq}}$ & $36.66 \mathrm{az}$ & $32.63_{\mathrm{q}-\mathrm{y}}$ & $149.73_{\mathrm{r}-\mathrm{Z}}$ & $77.06_{\mathrm{a}-\mathrm{o}}$ & $102.16_{c-j}$ & $7.08 \mathrm{bcd}$ & $25.66_{\mathrm{s}-\mathrm{z}}$ & $1.96_{\mathrm{s}-\mathrm{v}}$ & $1.67_{\mathrm{w}-\mathrm{Z}}$ & $121_{\mathrm{t}-\mathrm{x}}$ \\
\hline HD96 & $25.2_{\mathrm{f}-\mathrm{s}}$ & $44.11_{\mathrm{vw}}$ & $46.5_{\mathrm{m}-\mathrm{r}}$ & $32.21_{\mathrm{r}-\mathrm{y}}$ & $151.15_{\mathrm{n}-\mathrm{z}}$ & $71.33_{\mathrm{g}-\mathrm{t}}$ & $98.16_{\mathrm{g}-\mathrm{s}}$ & $4.91_{t-y}$ & $24.5_{x y z}$ & $2.46_{\mathrm{i}-\mathrm{s}}$ & $2.19_{\mathrm{f}-\mathrm{p}}$ & $122.83_{\text {i-o }}$ \\
\hline HD116 & $18.81_{\text {s-y }}$ & $48.95 \mathrm{~h}-\mathrm{k}$ & $32.83 b$ & $2.20_{\mathrm{r}-\mathrm{y}}$ & $177.16_{\mathrm{g}-\mathrm{w}}$ & $76.75_{\mathrm{a}-\mathrm{p}}$ & & $3.91 \mathrm{bcd}$ & $25 \mathrm{u}-\mathrm{z}$ & $2.08_{\mathrm{p}-\mathrm{v}}$ & $1.77 \mathrm{t}-\mathrm{z}$ & $123 \mathrm{~h}-\mathrm{n}$ \\
\hline HD3 & & & & & & & & $7.16_{b c}$ & & & $1.89_{\mathrm{p}-\mathrm{z}}$ & $.16_{\text {cde }}$ \\
\hline HD22 & $19.20_{\mathrm{q}-\mathrm{y}}$ & $41.24_{\mathrm{abz}}$ & $39.66_{w-z}$ & $31.65 \mathrm{r}-\mathrm{z}$ & $171.72_{\mathrm{h}-\mathrm{y}}$ & $75.33_{\mathrm{c}-\mathrm{q}}$ & $90.16_{a-d}$ & $5 s_{-y}$ & $25.16_{\mathrm{t}-\mathrm{z}}$ & $2.24_{\mathrm{n}-\mathrm{u}}$ & $1.80_{\mathrm{r}-\mathrm{z}}$ & $123.33_{\mathrm{f}-\mathrm{l}}$ \\
\hline HD35 & $23.11_{\text {h-w }}$ & $34.08 \mathrm{l}$ & $45_{\mathrm{p}-\mathrm{u}}$ & $31.58_{\mathrm{s}-\mathrm{z}}$ & $209.98_{\mathrm{d}-\mathrm{k}}$ & $73.05_{\mathrm{f}-\mathrm{r}}$ & $103_{b-g}$ & $6.63_{\mathrm{c}-\mathrm{g}}$ & $27.16_{n-v}$ & $2.45_{\mathrm{i}-\mathrm{s}}$ & $1.57 \mathrm{acyz}$ & $121.16_{\mathrm{s}-\mathrm{w}}$ \\
\hline HD130 & $20,29 \mathrm{~m}-\mathrm{y}$ & $49.35_{\mathrm{gh}}$ & $48.66_{\mathrm{i}-\mathrm{n}}$ & $31.40_{\mathrm{s}-\mathrm{z}}$ & $123.21_{\mathrm{abyz}}$ & $71.23 \mathrm{i}-\mathrm{t}$ & $100.16_{d-n}$ & $5.16_{\mathrm{r}-\mathrm{w}}$ & $27.61-\mathrm{t}$ & $2.55 \mathrm{~h}-\mathrm{r}$ & $2.07_{\mathrm{j}-\mathrm{u}}$ & $123 \mathrm{~h}-\mathrm{n}$ \\
\hline HD122 & $26,55_{\mathrm{d}-\mathrm{m}}$ & $45.17_{\mathrm{tu}}$ & $39.83_{w-z}$ & $1.23 \mathrm{t}-\mathrm{z}$ & $156.31 \mathrm{~m}-\mathrm{z}$ & $77.82 \mathrm{a}-\mathrm{n}$ & 91 & $4.55_{\mathrm{w}-\mathrm{z}}$ & $30.833_{c-j}$ & $2.85 \mathrm{c}-\mathrm{k}$ & $2.71 b c$ & $125.5 \mathrm{a}-\mathrm{b}$ \\
\hline 117 & & $50.54 \mathrm{e}$ & $37.16_{a z}$ & 31 & 16 & $73.94^{\mathrm{e}-\mathrm{r}}$ & & & $33.3_{\mathrm{abc}}$ & 2.63 & $1.96 \mathrm{~m}-\mathrm{x}$ & $123.6_{e-j}$ \\
\hline HD133 & $18,78_{\text {s-y }}$ & $43.09 x$ & $45.66 n-t$ & $31.12^{\mathrm{t}-\mathrm{z}}$ & $139.41^{\mathrm{t}-\mathrm{z}}$ & $69.70^{\mathrm{k}-\mathrm{u}}$ & $106.33^{\mathrm{ab}}$ & $4.65^{\mathrm{u}-\mathrm{z}}$ & $27^{0-w}$ & $2.22^{\mathrm{n}-\mathrm{u}}$ & $1.79^{\mathrm{s}-\mathrm{z}}$ & $124.16_{c-g}$ \\
\hline HD132 & $21,74_{\text {i-y }}$ & $37.22 \mathrm{hij}$ & $42_{\mathrm{u}-\mathrm{x}}$ & $31.11_{\mathrm{t}-\mathrm{z}}$ & $217.68 \mathrm{c}-\mathrm{i}$ & $76.37_{\mathrm{b}-\mathrm{p}}$ & $97.83_{\mathrm{h}-\mathrm{t}}$ & $4.91_{t-y}$ & $29.16_{\text {i-p }}$ & $2.30_{1-u}$ & $1.87_{\mathrm{p}-\mathrm{z}}$ & $124.33_{\mathrm{c}-\mathrm{f}}$ \\
\hline HD47 & $17.97_{\mathrm{v}-\mathrm{y}}$ & $40.69 \mathrm{bcd}$ & $42.83_{\mathrm{t}-\mathrm{w}}$ & $31.10_{\mathrm{u}-\mathrm{z}}$ & $136.68_{\mathrm{u}-\mathrm{z}}$ & & $95.66_{\mathrm{m}-\mathrm{y}}$ & & $27.33_{\mathrm{m}-\mathrm{u}}$ & $2.23_{\mathrm{n}-\mathrm{u}}$ & $1.79_{\mathrm{s}-\mathrm{z}}$ & $120.5_{\mathrm{v}-\mathrm{y}}$ \\
\hline EXP & $25.88_{\mathrm{e}-\mathrm{p}}$ & $40.81_{\mathrm{ab}}$ & $35.66_{a b}$ & $30.89 \mathrm{u}-\mathrm{z}$ & $260.88_{\mathrm{abc}}$ & $69.74_{\mathrm{k}-\mathrm{u}}$ & $94.83_{0-\mathrm{z}}$ & & $30_{\mathrm{F}-1}$ & $1.94_{\mathrm{s}-\mathrm{v}}$ & $1.54 \mathrm{acbz}$ & $125.16_{\mathrm{abc}}$ \\
\hline HD125 & 20,82 k-y & $36.05_{\mathrm{k}}$ & $44_{\mathrm{rv}}$ & $30.86_{\mathrm{u}-\mathrm{z}}$ & $205.3 \mathrm{~d}-\mathrm{m}$ & $68.85_{\mathrm{m}-\mathrm{v}}$ & & $5.5 \mathrm{~m}-\mathrm{t}$ & $33.16_{\mathrm{a}-\mathrm{d}}$ & $1.79_{\mathrm{uv}}$ & $1.52_{\mathrm{abc}}$ & $122.5_{\mathrm{k}-\mathrm{q}}$ \\
\hline HD8 & $25.83 \mathrm{e}-\mathrm{p}$ & $34.36 \mathrm{I}$ & $44.83 \mathrm{q}-\mathrm{u}$ & $30.61_{u-z}$ & $196.94 \mathrm{e}-\mathrm{p}$ & $69.97_{\mathrm{k}-\mathrm{u}}$ & $96.33 \mathrm{k}-\mathrm{y}$ & $5.16 \mathrm{r}-\mathrm{w}$ & $29.5_{\mathrm{h}-\mathrm{o}}$ & $2.62 \mathrm{e}-\mathrm{o}$ & $2.15_{\mathrm{g}-\mathrm{r}}$ & $122.83_{\mathrm{i}-\mathrm{o}}$ \\
\hline HD77 & $21.15_{\mathrm{j}-\mathrm{y}}$ & $40.91_{\mathrm{ab}}$ & $42_{\mathrm{u}-\mathrm{x}}$ & $30.46_{\mathrm{u}-\mathrm{z}}$ & $179.88_{\mathrm{f}-\mathrm{w}}$ & $71.50_{\mathrm{g}-\mathrm{t}}$ & $89.83 \mathrm{bcd}$ & $4.83 \mathrm{t}-\mathrm{z}$ & $24.83_{\mathrm{V}-\mathrm{z}}$ & $2.21_{\mathrm{n}-\mathrm{u}}$ & $1.78_{\mathrm{s}-\mathrm{z}}$ & $122.33 \mathrm{l}-\mathrm{r}$ \\
\hline HD48 & $23.61_{\mathrm{g}-\mathrm{v}}$ & $37.95_{\mathrm{h}}$ & $54.83_{\mathrm{a}-\mathrm{e}}$ & $30.28_{\mathrm{u}-\mathrm{z}}$ & $170.28_{\mathrm{i}-\mathrm{y}}$ & $70.02_{\mathrm{j}-\mathrm{u}}$ & $93.16_{\mathrm{s}-\mathrm{z}}$ & $5.71_{\mathrm{k}-\mathrm{s}}$ & $22.83_{\mathrm{ab}}$ & $2.62 \mathrm{e}-\mathrm{o}$ & $2.20_{\mathrm{f}-\mathrm{p}}$ & $123_{\mathrm{h}-\mathrm{n}}$ \\
\hline HD 120 & & $47.7_{\mathrm{mno}}$ & $46.831-\mathrm{r}$ & & $142.22_{\mathrm{s}-\mathrm{z}}$ & $76.40_{\mathrm{b}-\mathrm{p}}$ & $98.16_{\mathrm{g}-\mathrm{s}}$ & $6_{\mathrm{h}-\mathrm{o}}$ & & $2.3 \mathrm{l}-\mathrm{u}$ & $1.93 \mathrm{n}-\mathrm{x}$ & $123.83_{\mathrm{e}-\mathrm{i}}$ \\
\hline EXI & $27.09 \mathrm{~d}-1$ & $37.10_{\mathrm{ij}}$ & $51_{\mathrm{f}-\mathrm{j}}$ & $29.56_{\mathrm{v}-\mathrm{Z}}$ & $170_{\text {i-y }}$ & $59.5 \mathrm{vWx}$ & & $6.5_{c-j}$ & $27.51_{-u}$ & $1.95_{\mathrm{s}-\mathrm{v}}$ & $1.74 \mathrm{u}-\mathrm{z}$ & $123.16_{\mathrm{g}-\mathrm{m}}$ \\
\hline HD34 & $16.68_{x y}$ & $45.83_{\mathrm{rst}}$ & $44_{r-v}$ & $29.56_{\mathrm{v}-\mathrm{z}}$ & $137.18_{\mathrm{u}-\mathrm{z}}$ & $78.66_{a-k}$ & $94.5_{\mathrm{p}-\mathrm{z}}$ & $5.91_{\mathrm{h}-\mathrm{p}}$ & $33.83_{\mathrm{ab}}$ & $2.81_{\mathrm{c}-\mathrm{l}}$ & $2.16_{\mathrm{g}-\mathrm{q}}$ & $122_{\mathrm{n}-\mathrm{s}}$ \\
\hline HD134 & $19,05_{\mathrm{r}-\mathrm{y}}$ & $46.57_{\mathrm{qrs}}$ & & $28.61_{\mathrm{w}-\mathrm{z}}$ & $143.85_{\mathrm{s}-\mathrm{z}}$ & $78.37 \mathrm{a}-\mathrm{m}$ & & $4.83 \mathrm{t}-\mathrm{z}$ & $27_{\text {o-w }}$ & $2.65_{\mathrm{e}-\mathrm{o}}$ & $2.32 \mathrm{e}-\mathrm{m}$ & $123.16_{\mathrm{g}-\mathrm{m}}$ \\
\hline PLAI & $18.32_{\mathrm{u}-\mathrm{y}}$ & $39.99_{\mathrm{c}-\mathrm{f}}$ & $51.66_{\mathrm{e}-\mathrm{i}}$ & $28.10_{\mathrm{w}-\mathrm{z}}$ & $135.35_{\mathrm{v}-\mathrm{z}}$ & $79.37_{a-j}$ & & $5.83_{\mathrm{i}-\mathrm{q}}$ & $30.66_{d-j}$ & $2.3_{1-u}$ & $1.9_{\mathrm{p}-\mathrm{z}}$ & $126_{a}$ \\
\hline & & & $44.83 \mathrm{q}-\mathrm{u}$ & 27.44 abyz & $128.9_{\mathrm{w}-\mathrm{z}}$ & $62.74 \mathrm{~s}-\mathrm{v}$ & $99.66 \mathrm{~d}-\mathrm{o}$ & 4.16abcdz & & $2.40_{\mathrm{j}-\mathrm{s}}$ & $2.09_{\mathrm{i}-\mathrm{u}}$ & $125 \mathrm{a}-\mathrm{d}$ \\
\hline HD 135 & $23,28 \mathrm{~h}-\mathrm{x}$ & $41.57_{\mathrm{ayz}}$ & $50.5 \mathrm{f}-\mathrm{k}$ & $27.39_{\text {abyz }}$ & 123.88 xyz & 77.38 a-o & $93.33_{\mathrm{s}-\mathrm{z}}$ & $5.5 \mathrm{~m}-\mathrm{t}$ & $30.83_{c-j}$ & $3.08 \mathrm{c}-\mathrm{g}$ & $2.46_{c-g}$ & $121.33_{\mathrm{r}-\mathrm{v}}$ \\
\hline HD49 & & $45.81_{\mathrm{st}}$ & $44.83_{\mathrm{r}-\mathrm{u}}$ & $25.66_{\mathrm{abz}}$ & $111.95 \mathrm{ab}$ & $70.02 \mathrm{j}-\mathrm{u}$ & $97.33_{\mathrm{i}-\mathrm{v}}$ & $5.41_{n-t}$ & $23.66_{a b y z}$ & $2.55_{\mathrm{h}-\mathrm{r}}$ & $2.20_{\mathrm{f}-\mathrm{p}}$ & $122.66_{\mathrm{j}-\mathrm{p}}$ \\
\hline HD74 & $17.00_{\mathrm{wxy}}$ & $39.26_{\mathrm{fg}}$ & $51.666_{-i}$ & $23.47_{\mathrm{ab}}$ & $101.64 \mathrm{~b}$ & $61.98_{\mathrm{t}-\mathrm{v}}$ & & & $29.66_{g-n}$ & $3.01_{\mathrm{c}-\mathrm{h}}$ & $2.45_{\mathrm{c}-\mathrm{i}}$ & $122_{\mathrm{n}-\mathrm{s}}$ \\
\hline HD80 & $21.95_{\mathrm{i}-\mathrm{x}}$ & $41.25_{\mathrm{abz}}$ & $38.5_{\mathrm{ayz}}$ & $22.43 b$ & $146.38_{\mathrm{s}-\mathrm{z}}$ & $69.061-u$ & & $6.16_{\mathrm{g}-\mathrm{m}}$ & $30.5_{e-j}$ & $2.20_{\mathrm{n}-\mathrm{u}}$ & $2.21_{\mathrm{f}-\mathrm{p}}$ & $118.16 \mathrm{bc}$ \\
\hline M A & 24.68 & 44.65 & 46.21 & 36.92 & 178.82 & 73.73 & 96.40 & 5.42 & 28.26 & 2.53 & 2.09 & 122.06 \\
\hline $\mathrm{CV}(\%)$ & 42.31 & 12.37 & 17.46 & 31.10 & 33.68 & 21.00 & 9.61 & 24.92 & 15.73 & 30.56 & 30.23 & 8.53 \\
\hline LSD & 6.49 & 0.76 & 3.25 & 6.23 & 50.33 & 9.55 & 5.11 & 0.72 & 2.53 & 0.53 & 0.35 & 1.01 \\
\hline
\end{tabular}

Note: In a column, numbers with same letter ( $)$ do not differ significantly at $\mathrm{p}=0.05$. Gen: Genotype, MA: Mean of accessions 
Table 5. Pearson's correlation coefficients

\begin{tabular}{|c|c|c|c|c|c|c|c|c|c|c|c|c|}
\hline Traits & HI & TGW & NGS & GY & SN & RWC & PH & SL & PL & SW & GWS & DHE \\
\hline $\begin{array}{l}\mathrm{HI} \\
\end{array}$ & 1 & & & & & & & & & & & \\
\hline TGW & 0.366 & 1 & & & & & & & & & & \\
\hline NGS & 0.078 & 0.028 & 1 & & & & & & & & & \\
\hline GY & 0.741 & 0.511 & 0.171 & 1 & & & & & & & & \\
\hline $\mathrm{SN}$ & 0.567 & -0.138 & -0.354 & 0.597 & 1 & & & & & & & \\
\hline RWC & 0.246 & 0.369 & -0.033 & 0.410 & 0.179 & 1 & & & & & & \\
\hline PH & 0.058 & 0.148 & -0.037 & 0.136 & 0.016 & 0.185 & 1 & & & & & \\
\hline SL & 0.172 & -0.142 & 0.053 & 0.081 & 0.092 & 0.067 & -0.013 & 1 & & & & \\
\hline PL & 0.107 & 0.064 & -0.075 & -0.070 & -0.061 & 0.036 & 0.405 & 0.091 & 1 & & & \\
\hline SW & 0.036 & 0.316 & 0.560 & 0.099 & -0.393 & 0.069 & $-0,041$ & -0.136 & -0.015 & 1 & & \\
\hline KWS & 0.049 & 0.321 & 0.576 & 0.028 & -0.478 & 0.045 & -0.035 & -0.253 & 0.030 & 0.875 & 1 & \\
\hline DHE & -0.315 & -0.395 & -0.099 & -0.465 & -0.113 & -0.435 & -0.044 & -0.260 & -0.022 & -0.006 & 0.052 & 1 \\
\hline
\end{tabular}

Note: The values in bold are different from zero to a level of significance alpha $=0.05$

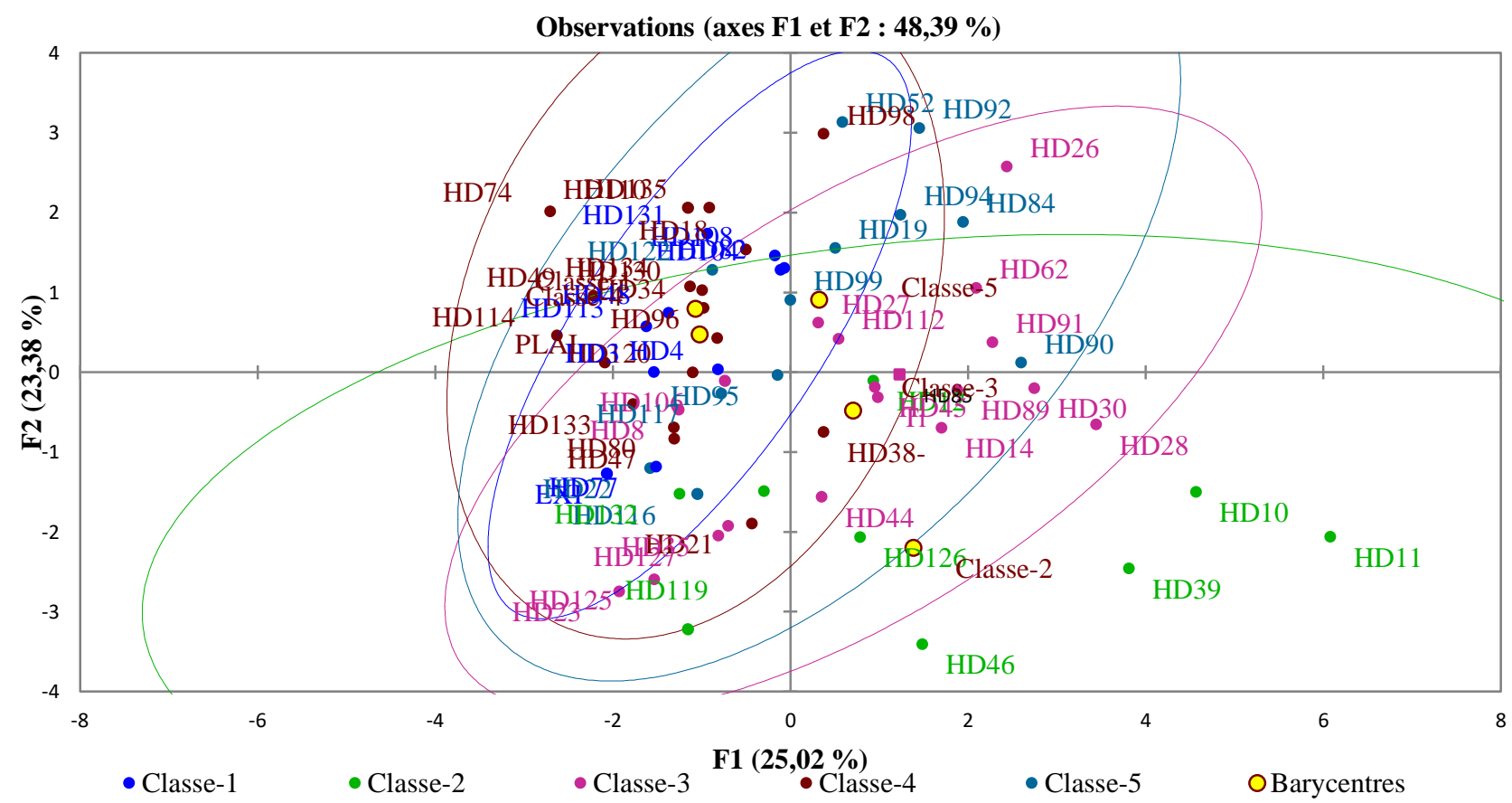

Figure 1. Scatter diagram with the distribution of sixty-six double-haploid (DH) barley line and four parents according to the first two principal components (PC1 and PC2)

The first group includes 38 genotypes, where the first subgroup contains 21doubled haploid (DHs) barley lines (HD15, HD91, HD52, HD45, HD112, HD90, HD89, HD94,HD26,HD77, HD22, HD116, HD95, HD108, HD4, HD104, HD82, HD48, HD131, HD113, HD3 and the variety Exito (Exi).The second subgroup of the first group consisted of 17 double-haploid (HD) barley lines HD27, HD23, HD132, HD127, HD35, HD8,HD125, HD126, HD119, HD44, HD12,HD30,HD14,HD62,HD28 and
Tichedrett (Ti) local variety. The second group consisted of the lines HD10, HD11, HD38, HD39, HD46, HD126 and HD119. The third group includes 26 genotypes, where the first subgroup contains 12 double haploid (DHs) barley lines HD134, HD120, HD47, HD34, HD135, HD133, HD114, HD49, HD130, HD18, HD74 and Plaisant (PLAI). The second subgroup of third group consisted the lines HD99. HD96, HD122, HD110, HD80, HD 117, HD21, HD 98, HD15, HD 84, HD92, HD19 and HD85. 


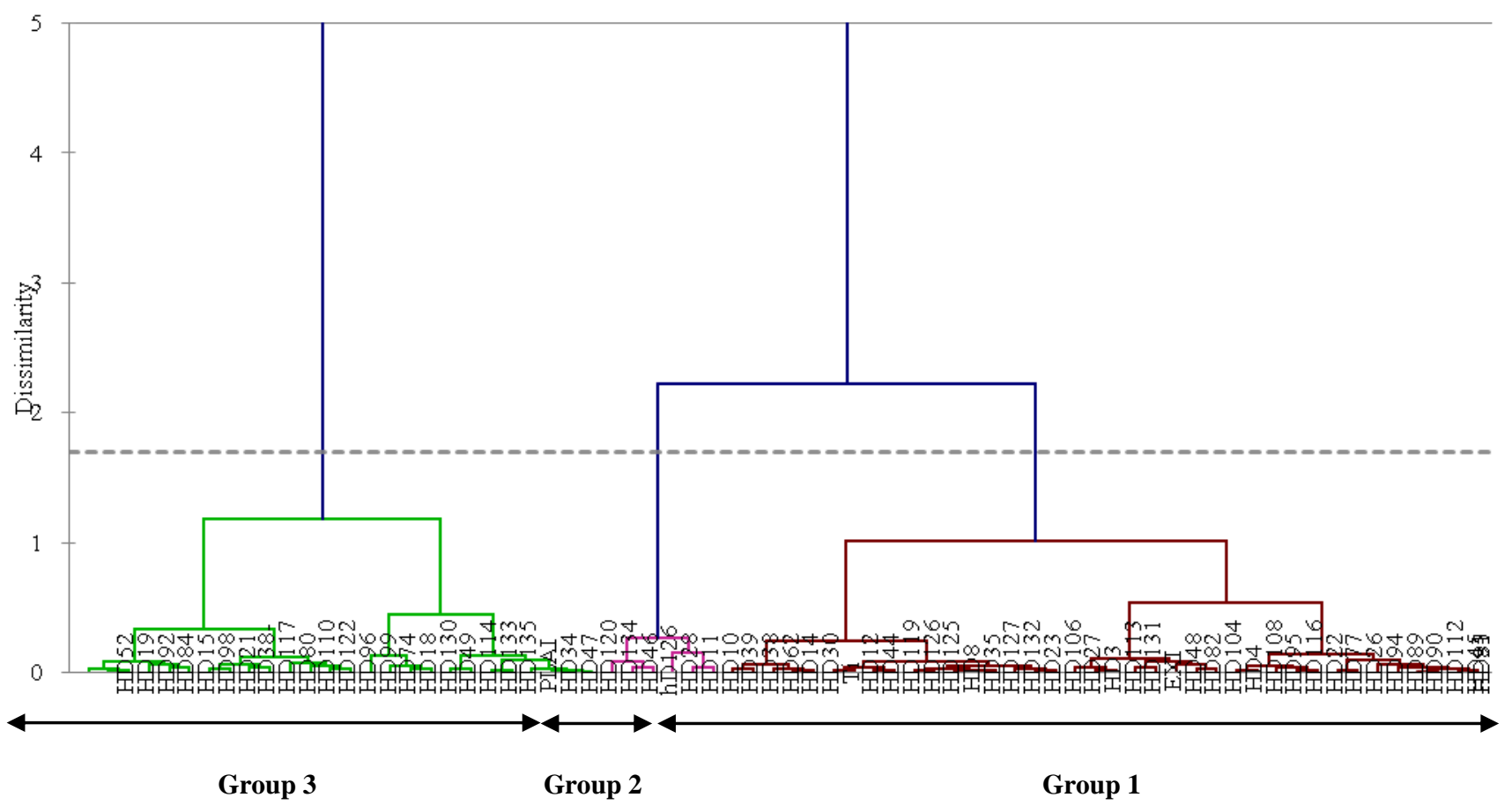

Figure 2. Dendrogram computed from the Euclidean distance for sixty-six double-haploid (DH) barley line and four parents

Table 6. Eigenvalues, individual and cumulative percentage variations, and Eigenvector explained by four vectors explained by four principal components based on agronomic traits in sixtysix double-haploid (DH) barley line and four parents evaluated in two locations (Constantine and Setif)

\begin{tabular}{|c|c|c|c|c|}
\hline Parameters & PC1 & PC2 & PC3 & PC4 \\
\hline Eigen value & 3.009 & 2.803 & 1.456 & 1.176 \\
\hline$\%$ of variance & 25.077 & 23.358 & 12.132 & 9.798 \\
\hline Cumulative $\%$ & 25.077 & 48.434 & 60.567 & 70.364 \\
\hline Characters & \multicolumn{4}{|c|}{ Eigenvector } \\
\hline HI & 0.797 & -0.106 & -0.153 & -0.031 \\
\hline TGW & 0.625 & 0.367 & 0.181 & -0.281 \\
\hline NGS & 0.111 & 0.695 & -0.212 & 0.377 \\
\hline GY & 0.909 & -0.044 & -0.195 & -0.11 \\
\hline $\mathrm{SN}$ & 0.486 & -0.666 & -0.256 & -0.197 \\
\hline RWC & 0.614 & 0.014 & 0.178 & -0.026 \\
\hline $\mathrm{PH}$ & 0.215 & -0.035 & 0.771 & -0.065 \\
\hline SL & 0.174 & -0.254 & -0.021 & 0.064 \\
\hline PL & 0.082 & -0.011 & 0.786 & 0.153 \\
\hline SW & 0.134 & 0.893 & -0.074 & 0.006 \\
\hline GWS & 0.079 & 0.93 & -0.022 & -0.081 \\
\hline DHE & -0.651 & 0.012 & -0.015 & $-0,349$ \\
\hline
\end{tabular}

\section{Discussion}

Sixty-six doubled-haploid (DHs) barley lines derived from $\mathrm{F}_{2}$ plants of 3 bi-parental single crosses, between the local variety (Tichdrett) and three introduction varieties (Express, Plaisant, and Exito) were evaluated in 2017 using randomized block design with three replication at two locations under rainfed conditions of semi-arid zone in Algerian. Pooled analysis of variance across environments indicated the presence of highly significant genotype effect for all traits except leaf area (LA) and a highly significant environment effect for all characters studied, except spikes number per $\mathrm{m}^{2}(\mathrm{SN})$ and Awns length (AL).

The results of ANOVA for quantitative traits indicated the influence of climatic conditions on phenological, and traits related to yield. Setif has a semi-arid climate, with winter rainfall (annual rainfall of $334.7 \mathrm{~mm}$ ) as well as in Constantine location (annual rainfall of $403 \mathrm{~mm}$. According to Ramla et al. (2015), barley production is mainly located in the highland semi-arid agro-climatic zone (300-400 mm rainfall) characterized by variable and severe climatic conditions. These environmental factors appear to have substantial impact on the phonological and yield components of barley. The main limitation to higher yield in the Mediterranean environment is water availability (Rizza et al. 2004); therefore, superior germplasm under the water-limited growing conditions may carry some positive drought tolerance traits. Abundant research on drought tolerance of crops indicates that different mechanisms may be relevant at different productivity levels (Cattivelli et al. 2008).

A wide range of variations was found among the DHs lines for all the traits. Moreover, the HDs lines expressed better performance for seed production in Constantine. At Setif earlier heading and a higher vegetative development were observed. Generally, DHs lines grown in Constantine produced longer spikes with higher number of grains per spike (NGS) and superior thousand-grain weight (TGW), than those grown in Setif. In addition, the mean grain yield 
(GY) and Harvest index (HI) were superior at Constantine compared to Setif. Whereas, Results indicated also that more important DHs lines proportion were associated with leaf relative water content (RWC) and plant height (PHT) in Constantine region. These results would suggest that high temperature and rainfall distribution during the vegetative growth are among the factors that enhanced the expression of these traits. The mode of inheritance of grain yield and most agronomic traits is complex, governed by several genes and influenced by environmental conditions (Falconer 1981; Peighambari et al. 2005). A number of genes control these traits, and in general, they react in the same manner according to environmental conditions (Bellatreche 2017).

Genotypes x location interaction was height significant for all traits, except specific weight leaf (SWL) and leaf area (LA). These results agree much with those found bay Drikvand et al. (2012) where traits contributing to the most variance concerned awn length, plant height, grain yield, grain number per spike, peduncle length, spike length, and 1000-grain weight. Many of these traits have also contributed to the genetic differentiation among accessions of barley (Setotaw et al. 2010). Previous studies have also reported significant $\mathrm{G} \times \mathrm{E}$ effect for different agronomic characters in barley populations (Teulat et al. 2001; Pillen et al. 2003; Peighambari et al. 2005; Von Korff et al. 2006; Chand et al. 2008; Schmalenbach et al. 2009).

Genotypes $\mathrm{x}$ environment interactions are important sources of variation in any crop and the term stability is sometimes used to characterize a genotype, which shows a relatively constant yield, independent of changing environmental conditions (Becker and Leon 1988).

Significant differences were observed among barley cultivars for grain yield, 1000-grain weight, plant height and heading date (Mut et al. 2010). The significant estimates of $\mathrm{G} \times \mathrm{E}$ interaction indicated that the characters were unstable and may considerably fluctuate with change in environments (Chand et al. 2008). These results indicated the presence of variability among genotypes as well as environments under which the experiments were conducted. Therefore, an understanding of genotypes $\mathrm{x}$ environments interaction provides valid insights to words the selection of new stable genotypes in the diversified environmental conditions prevailing in a region.

Variability of quantitative traits of any crop is influenced by genetic factors, environmental factors, and their interaction. Uniformity of individuals and stability of quantitative traits are major requirements for the development of improved varieties and their release. Since the preservation of broad genetic base of landraces could be much appreciated, then the study of variability of quantitative traits becomes increasingly important. In our experiment, the most variable trait was harvest index (HA) followed by yield components: spike number per $\mathrm{m}^{2}(\mathrm{SN})$, grain weight per spike (GWS), gains yield of grains (GY) with respective coefficient of variation values of $42.33 \%$, $33.68 \%, 30.23 \%$, and $31.10 \%$. Such a strong variability was caused by the fact that grain yield is a complex trait controlled by a polygenetic system and is strongly influenced by environmental factors. Darwinkel (1978) in
Chalak et al. (2015) reported similar pattern of variability in winter wheat grown at the Netherlands a wide range of plant population densities.

Days to heading expressed the lowest variability with a coefficient of variation of $8.53 \%$, followed by plant height $(9.6 \%)$ and peduncle length $(15.73 \%)$. These results match with the findings of Singh (2011), who reported that days to heading were the most stable traits, whereas yield and yield components were noticed for strong phenotypic and genotypic variability about $30 \%$.

The genetic correlations among the twelve agronomic traits were estimated from data combined across environments table. Significant positive correlation, ranging from 0.45 to 0.87 , were observation among number of grains per spike, thousand grains weight, spikes number per $\mathrm{m}^{2}$, grain yield and harvest index. Similar results have been reported by Peighambari et al. (2005) in Iran and Rajiv and Yashasvita (2018) in India. Many workers have also reported similar positive and significant association of grain yield with Spikes number per $\mathrm{m}^{2}$ (Akdeniz et al. 2004; Ataei 2006), number of grains per spike (Drikvant et al. 2011). Thousand-grain weight and with harvest index (Khajavi et al. 2014; Kumar et al. 2014).The same finding for grain yield and plant height were reported by other studies (Samarrai et al. 1987; Kisana et al. 1999; Bhutta et al. 2005). Akdeniz et al. (2004) observed positive and significant correlations between grain yield and yield components such as plant height, spike length and spike number per $\mathrm{m}^{2}$ but found negative and non-significant correlations between grain yield and grains number per spike. Ataei (2006), reported positive and significant correlations of grain yield with spike number per $\mathrm{m}^{2}$ and thousand grains weight.

Principal component analysis was used to observe the general pattern for variation of traits. The first four principal components with eigenvalues greater than unity (1) together extracted about $72 \%$ of the total variation. According to Johnson and Wichern (2002), based on the Eigenvalues and vectors, it is possible to indicate which traits are mainly responsible to explain the variation. Accordingly, the first principal components, which contributed about $48.42 \%$ of total variation, were due to harvest index, leaf relative water content, thousand grains weight, grain yield and number of days to heading, respectively (Table 4). Similarly, about $23.35 \%$ of the variation, accounted for by the second principal component, was due to contributions of number of grains per spike, spike length, spike weight, grain weight per spike and spikes number.

On the other hand, the third principal component, which explained about $12.13 \%$ of the variation, mainly planted height followed by Peduncle length, while the fourth principal component showed $10 \%$ of total variation with greater loading from spike length and number of grains per spike. In the work of Abebe et al. (2010) for Ethiopian barleys in relation to geographic regions and altitudes, the first three principal components (PCs), with eigenvalues greater than unity, explained about $73 \%$ of the total variation among accessions for the nine quantitative traits. Hence, even if the genotypes and the number of traits used 
vary, the value of the first three principal components greater than unity shows a better percentage in this study than the genotypes investigated by Abebe et al. (2010). According to the study of Zaheer et al. (2008) in Pakistan, the variation studied through Principal Component Analysis revealed that five principal components having greater than 1eigenvalues contributed $83.40 \%$ of the total variation.

Although the cluster analysis grouped, barley accessions with greater morphological similarity, the dendrogram of the evaluated sixty-six double-haploid (DHs) barley lines and four parents genotypes grouped into three cluster groups. Konichi et al. (1993) in Bhutan, sixrow barley genotypes investigated were also classified into three groups. It is the same for a study by DimitrovaDoneva et al. (2014).

The first group includes 38 genotypes which was $54.28 \%$ of the total experimental materials, where the first subgroup contains 21double-haploid (DHs) barley lines, among these lines: HD77, HD4, HD104, HD82, HD48, HD131, HD113, HD3 and the variety Exito (Exi), characterized by the highest number of grains per spike, grain weight per spike and spike weight. The second subgroup of the first group consisted of 17 double-haploid (HD) barley lines, among which: HD27, HD23, HD132, HD127, HD35, HD8, HD125, HD44, HD28 and a local variety Tichedrett $(\mathrm{Ti})$, characterized by the highest value of spike length and spikes number per $\mathrm{m}^{2}$. The second group consisted of the lines HD10, HD11, HD38, HD39, HD46, HD126 and HD119, characterized by the highest values of harvest index, thousand grains weight, and grain yield.

The third group includes 25 genotypes, $35.71 \%$ of the population, where the first subgroup contains 12 double haploids (DHs) barley lines HD134, HD120, HD47, HD34, HD135, HD133, HD114, HD49, HD130, HD18, HD74, and the introduced variety Plaisant (PLAI), characterized by long number of days to heading. The second subgroup of third group consisted of 13 lines, among them: HD122, HD117, HD84, HD92, HD19, and HD95, characterized by long plant height and peduncle length. The confirmation of these results and the study of other agronomic parameters and those related to the biotic and abiotic stresses will be used for better identifying this germplasm and better directing works of genetic improvement.

\section{REFERENCES}

Abebe TD, Bauer AM, Leon J. 2010. Morphological diversity of Ethiopian barleys (Hordeum vulgare L.) in relation to geographic regions and altitudes. Hereditas 147: 154-164.

Akdeniz H, Keskin B, Y1lmaz I, Oral E. 2004. Research on yield and yield components of some barley cultivars. J Agric Sci 14: 119-125.

Alberts MJA. 2004. Comparison of Statistical Methods to Describe Genotype-Environment Interaction and Yield Stability in MultiLocation Maize Trials. [Thesis]. University of the Free State, South Africa.

Araus JL, Amaro T, Voltas J, Nakkoul H, Nachit MM. 1998. Chlorophyll fluorescence as a selection criterion for grain yield in durum wheat under Mediterranean conditions. Field Crops Res 55 (3): 209-223.

Ataei M. 2006. Path analysis of barley (Hordeum vulgare L.) yield. Ankara Univ Fac Agric J Agric Sci 12: 227-232.
Becker HC, Leon J. 1988. Stability analysis in plant breeding. Plant Breed 101: 1-23.

Bedasa M, Berhane L, Tadesse D. 2015. Morphological diversity and association of traits in Ethiopian food barley (Hordeum vulgare L). Landraces in relation to regions of origin and altitudes. J Plant Breed Crop Sci 7 (2): 44-54.

Belkherchouche H, Fellah S, Bouzerzour H, Benmahammed A, Chellal N. 2009. Vigor growth, translocation and grain yield of durum wheat (Triticum durum Desf.) under semi-arid conditions. Courrier du savoir 9: 17-24. [French]

Bellatreche A, Moustafa Yassine Mahdad M Y, Kaouadji Z, Gaouar SBS. 2017. Agro-morphological diversity of some accessions of bread wheat (Triticum aestivum) in western Algeria. Biodiversitas 18: 409415.

Bhutta WM, Barley T, Ibrahim M. 2005. Path-coefficient analysis of some quantitative characters in husked barley. Ser Biol 17: 65-70.

Cattivelli L, Rizza F, Badeck FW, Mazzucotelli E, Mastrangelo AM, Francia E, Mare C, Tondelli A, Stanca AM. 2008. Drought tolerance improvement in crop plants, an integrated view from breeding to genomics. Field Crops Res 105: 1-14.

Ceccarelli S, Grando S, Baum M, Udupa SM. 2004. Breeding for Drought Resistance in a Changing Climate. In: Rao SC, Ryan J (eds.). Challenges and Strategies of Dryland Agriculture. CSSA Special Publication No. 32, CSSA/ASA, Madison, Wisconsin.

Ceccarelli S, Grando S, Capettini F. 2011. Barley Breeding History, Progress, Objectives and Technology-Near East, North and East Africa and Latin America. In: Ulrich SE (ed.). Barley: Production, Improvement and Uses, Wiley, New York.

Ceccarelli S. 1996. Adaptation to low /high input cultivation. Euphytica 92: 203-214.

Ceccarelli S, Grando S, Impiglia A. 1998. Choice of selection strategy in breeding barley for stress environments. Euphytica 103: 307-318.

Chalak L, Mzid R, Rizk W, Hmedeh H, Kabalan K, BreidyJ, Hamadeh B, Machlab H, Rizk H, Samih Elhajj S. 2015. Performance of 50 Lebanese barley landraces (Hordeum vulgare L. subsp. vulgare) in two locations under rainfed conditions. Ann Agric Sci 60 (2): 325334.

Chand N, Vishwakarma S.R, Verma, O.P, Kumar M. (2008. Phenotypic stability of elite barley lines over heterogeous environments. Barley Genetics Newslett 38: 14-17.

Chennafi H, Bouzerzour H, et Saci A. (2008. Improvement of rainfed agriculture in semi-arid environment. In: Proceedings of the National Seminar on Constraints to the Production of Durum Wheat in Algeria. Université Chlef, le 29 et 30 Novembre 2008, [French]

Derbal N. 2015. Study of the Spatio-Temporal Variation of Certain Technological Characteristics of Some Varieties of Durum Wheat Grown in Algeria. [Dissertation]. Université Badji, Mokhtar, Annaba. [French]

Dimitrova-Doneva D, Valcheva D, Vulchev D, Dylogerova B, Gotcheva M, Popova T. 2014. Evaluation of grain yield in advanced lines tworowed winter barley. Agric Sci Technol 6 (2): 165-169.

Drikvand R, Hossinpor T, Samiei K. 2011. Plant Coefficient Analysis in Hull less Barley under rainfed conditions Austr J Basic Appl Sci 5 (12): 277-279.

Drikvand R, Salahvarzi E, Salahvarzi A, Hossinpour T. 2012. Study of genetic diversity among rainfed barley genotypes using ISJ markers and morphological traits. J Agric Sci 4 (9): 137-144.

Falconer DS. 1981. Introduction to Quantitative Genetics. 2 $2^{\text {nd }}$ ed, Longman Group Ltd., London.

FAOSTAT. 2015. Food and Agriculture Organization (FAO) of the United Nations, Rome, Italy.

Gomez-Pando LR, Timenez-Davalos J, Eguiluz de la Barra A, AguilarCastellanos E, Falconi-Palomino J, Ibanez-Tremolada M. 2009. Filed performance of new in vitro androgenesis derived double haploids of barley. Euphytica 166: 269-276.

Johnson RA, Wichern DW. 2002. Applied Multivariate Statistical Analysis. Prentice Hall, New Jersey.

Khajavi A, Aharizad S, Ahmadizadeh M. 2014. Genetic diversity of promising lines of barley based on pheno-morphological traits in Ardabil area. I J Adv Biol Biomed Res 2 (2): 456-462.

Khaldoun A, Chery J, Monneveux P. 1990. Study of rooting characteristics and their role in water deficit adaptation in barley (Hordeum vulgare L). Agronomie EDP Sci 10 (5): 369-379. [French]

Kisana NS, Tahir M, Mujahid MY, Ahmed I, Majid A, Mustafa SZ, Ahmed Z. 1999. Variability and relationship between morpho- 
phenological traits and grain yield in winter and facultative barley under stress environments. Pak J Biol Sci 2: 767-771.

Konichi T, Yano Y, Fukushima Y. 1993. Genetic variation in barley landraces from Bhutan. Genet Resour Crop Ev 40: 33-38.

Kribaa M, Hallaire V, Curmi P, Lahmar R. 2001. Effects of various cultivation methods on the structure and hydraulic properties of soil in semi-arid climate. Soil Tillage Res 60: 43-53.

Kumar V, Kumar R Verma PS, Verma A, Sharma I. 2013 b. Recent trend in breeder seed production of barley (Hordeum vulgare L.) in India. Indian J Agric Sci 83: 576-578.

Kumar V, Rathore A, Kharub AS, Kumar D, Sharma I. 2014. GGE biplot analysis of multi-locational yield trials and identification of representative environments for barley (Hordeum vulgare L.) in India. Res Crops 15: 871-875.

Ma H, Busch R. H, Riera-Lizarazu O, Rines H. W, Dill-Macky R.1999. Agronomic performance of lines derived from anther culture, maize pollination and single-seed descent in a spring wheat cross. Theor Appl Genet 99: 432-436.

MARD. 2014. Ministry of Agriculture and Rural Development. Série B, Statistiques Agricoles. DSASI.

Mut Z, Gülümser A, Sirat A. 2010. Comparison of stability statistics for yield in barley (Hordeum vulgare L.). Afr J Biotechnol 9 (11): 1610 1618.

Pal D, Kumar S, Verma RPS. 2012. Pusa Losar (BHS 380)-the first dualpurpose barley variety for northern hills of India. Indian J Agric Sci 82: $164-165$

Pask AJD, Pietragalla J, Mullan DM, Reynolds MP. 2012. Physiological Breeding II: A field guide to wheat phenotyping. CIMMYT, Mexico, D.F.

Peighambari SA, Samadi BY, Nabipour A, Charmet G, Srrafi A. 2005 QTL analysis for agronomic traits in a barley doubled haploid population grown in Iran. Plant Sci 169: 1008-1013.

Pillen K, Zacharias A, Léon J. 2003. Advanced backcross QTL analysis in barley (Hordeum vulgare L.). Theor Appl Genet 107: 340-352.

Rahal-Bouziane H, Berrkani S, Merdas S, Nait Merzoug S, Abdelguerfi A. 2015. Genetic diversity of traditional genotypes of barley (Hordeum vulgare $\mathrm{L}$ ) in Algeria by phenomorphological and agronomic traits. Afr J Agric Res 10 (31): 3041-3048.

Rajiv KSR, Yashasvita C. 2018. Correlation analysis of grains yield and important agronomic traits in six-rowed barley (Hordeum Vulgare L.) in Agra Region, Intl J Sci Res 7 (3): 1836-1840.

Ramla D, Yakhou M. S Bilek N, Hamou M, Hhannachi A, Aissa A, Mekliche-Hanifi L. 2016. Grain yield stability analysis of barley doubled haploid lines in Algerian semi-arid zones. Asian J. Crop Sci 8: $43-51$.
Ramla D, Yakhou S, Hamou M, Haninfi-Mekliche L. 2017. Evaluation of the performance of some barley-doubled haploids (Hordeum vulgare L.) and identification of transgressive lines in an Algerian semi-arid zone. Nature \& Technology. http: //www.univ-chlef.dz/revuenatec. [French].

Rizza F, Badeck F.W, Cattivelli L, Lidestri O, Di Fonzo N, Stanca A.M 2004. Use of a water stress index to identify barley genotypes adapted to rainfed and irrigated conditions. Crop Sci. 44: 2127-2137.

Samarrai SM, Seyam SM, Mian HR, Dafie AA. 1987. Growth periods, harvest index and grain yield relationships in barley. Rachis Barley Wheat Newslett 6: 21-24.

Sameri M, Takeda K, Komatsuda T. 2006. Quantitative trait loci controlling agronomic traits in recombinant inbred lines from a cross of oriental-and occidental-type barley cultivars. Breed Sci 56: 242252.

SAS Institute Inc. 2015. SAS/IML® 14.1 User's Guide. SAS Institute Inc., Cary, NC.

Schmalenbach I, Léon J, Pillen K. 2009. Identification and verification of QTLs for agronomic traits using wild barley introgression lines. Theor Appl Genet 118: 483-497.

Setotaw TA, Dias Lads, Missio RF. 2010. Genetic divergence among barley accessions from Ethiopia. Crop. Breed. Appl. Biotechnol. 10: 116-123.

Shahinnia F, Rezai A, Sayed-Tabatabaei BE, Komatsuda T, Mohammadi SA. 2006. QTL mapping of heading date and plant height in barley cross 'Azumamugi' $\times$ 'Kanto Nakate Gold'. Iranian J Biotechnol 4: 88-94.

Singh AP. 2011. Genetic variability in two-rowed barley (Hordeum vulgare L.). Indian J Sci Res 2: 22-23.

Souri J, Dehghani H, Sabbaghza SH. 2005. Study of chickpea genotypes under water stress. Iran J Agric Sci 36: 1517-1527.

Teulat B, Mareh O, Souyris I, This D. 2001. QTL for agronomic traits from a Mediterranean barley progeny grown in several environments. Theor Appl Genet 103: 774-787.

Teulat-Merah B, Zoumarou W, Douimi R, Ben Salem M, Bahri H, This D. 1998. Study of drought tolerance in cereals: barley as a biological model QTL approach. In: Serge H (ed.). From Biological Models to Plant Improvement. Scientific Days of the AUF Network: Plant Biotechnology: Plant Improvement and Food Security, 7. Montpellier (FRA), 2000/07/03-05.

Von Korff M, Wang H, Léon J, Pillen K. 2006. AB-QTL analysis in spring barley: II. Detection of favorable exotic alleles for agronomic traits introgressed from wild barley (H. vulgare ssp. spontaneum). Theor Appl Genet 112.1221. 1231.

Zaheer A, Saif UA, Muhammad M, Muhammad Z, Muhammad SM. 2008. Genetic diversity for morpho-genetic traits in barley germplasm. Pak J Bot 40: 1217-1224. 